ARTICLE

Received 17 Aug 2014 | Accepted 10 Jun 2015 | Published 22 Jul 2015 DOl: 10.1038/ncomms8781

\title{
HDAC6 and RhoA are novel players in Abeta-driven disruption of neuronal polarity
}

\author{
Hanako Tsushima ${ }^{1, \star}$, Marco Emanuele ${ }^{1, \star}$, Alice Polenghi ${ }^{1}$, Alessandro Esposito ${ }^{1}$, Massimo Vassalli ${ }^{2}$, \\ Andrea Barberis ${ }^{1}$, Francesco Difato ${ }^{1} \&$ Evelina Chieregatti ${ }^{1}$
}

Maintenance of neuronal polarity and regulation of cytoskeletal dynamics are vital during development and to uphold synaptic activity in neuronal networks. Here we show that soluble $\beta$-amyloid (A $\beta$ ) disrupts actin and microtubule (MT) dynamics via activation of RhoA and inhibition of histone deacetylase 6 (HDAC6) in cultured hippocampal neurons. The contact of $A \beta$ with the extracellular membrane promotes RhoA activation, leading to growth cone collapse and neurite retraction, which might be responsible for hampered neuronal pathfinding and migration in Alzheimer's disease (AD). The inhibition of HDAC6 by $A \beta$ increases the level of heterodimeric acetylated tubulin and acetylated tau, both of which have been found altered in AD. We also find that the loss of HDAC6 activity perturbs the integrity of axon initial segment (AIS), resulting in mislocalization of ankyrin G and increased MT instability in the AIS concomitant with loss of polarized localization of tau and impairment of action potential firing.

\footnotetext{
${ }^{1}$ Department of Neuroscience and Brain Technologies, Istituto Italiano di Tecnologia, Genoa 16163, Italy. ${ }^{2}$ Institute of Biophysics, National Research Council of Italy, Genoa, Italy. ${ }^{*}$ These authors contributed equally to this work. Correspondence and requests for materials should be addressed to F.D. (email: francesco.difato@iit.it) or to E.C. (email: evelina.chieregatti@iit.it).
} 
E arly deficits in Alzheimer's disease (AD) are due to neurite degeneration, defects in axonal transport and alterations in the efficiency of neuronal transmission ${ }^{1,2}$. In $\mathrm{AD}$, synapse maintenance and reshaping of synaptic contacts are defective and result in early loss of synapses. In neurons, actin filaments are abundant in growth cones and dendritic spines, and disruption of actin turnover results in growth cone collapse ${ }^{3}$. In the mature nervous system, actin filaments form the basis of structural integrity in synapses ${ }^{4}$. On the basis of the critical role of actin in the formation of new synapses and the maintenance of synaptic integrity, we hypothesize that overproduction of beta-amyloid (A $\beta$ ) peptide, by inhibiting dynamic changes in actin polymerization, would lead to neurite retraction and synaptic dysfunction. Neurodegenerative stimuli can induce the formation of actin $\operatorname{rods}^{5}$ where $\mathrm{A} \beta$ has been shown to accumulate, and which block transport within neurites ${ }^{6}$. Retracted neurites and disorganized actin filaments are major components of senile plaques and neurofibrillary tangles (NFTs) ${ }^{7}$, which are hallmarks of $\mathrm{AD}$. $\mathrm{A} \beta$ and tau are also major components of extracellular senile plaques and intracellular NFTs, respectively, and increasing evidence indicates that the adverse effect of $A \beta$ is dependent on the presence of tau. It has been shown that $A \beta$ is unable to induce neurite degeneration in neurons derived from tau knockout mouse ${ }^{8}$, and that deletion or reduction of endogenous tau prevents the cognitive deficits observed in human amyloid precursor protein transgenic mice ${ }^{9}$. The transport deficit induced by $A \beta$ was also shown to be dependent on tau ${ }^{10}$ and exposure of cultured neurons to $A \beta$ leads to missorting of axonal tau into somatodendritic compartments, where it disrupts cytoskeletal components ${ }^{11,12}$. Alterations in neuronal polarity can lead not only to functional defects in axon potential propagation but also in the development of young neurons, with dysregulation of adult neurogenesis, a process that is specifically impaired in neurodegenerative diseases. However, the mechanisms and the targets through which $\mathrm{A} \beta$ affects cytoskeletal dynamics, and how these may influence axonal elongation or retraction, tau localization and neuronal polarity are still unclear. We exposed neurons both to soluble $A \beta_{1-42}$ peptide that is responsible for $A \beta$-induced toxicity ${ }^{13}$ and for the severity of $\mathrm{AD}^{14}$, to study MT dynamics, which are crucial in maintaining the integrity of AIS and the axonal localization of tau $^{15}$; and to beads coated with $A \beta$ to study the direct local effect of extracellular $\mathrm{A} \beta$ on actin remodelling, which regulate growth cone movements and axon growth.

Recently, tau was demonstrated to be acetylated in the microtubule (MT)-binding domain, a post-translational modification that impairs the binding of tau to MTs, and acetylated tau (ac-tau) was specifically found in tau aggregates in $\mathrm{AD}$ and related tauopathies ${ }^{16,17}$. Histone deacetylase 6 (HDAC6), a cytosolic member of class II HDACs ${ }^{18}$, is responsible for deacetylating both tubulin ${ }^{19}$ and tau ${ }^{16}$. Intriguingly, neurons in which HDAC6 were knocked down lose the axonal initial segment $(\mathrm{AIS})^{20}$, a trafficking barrier important for maintaining the polarized localization of axonal proteins, although the mechanism remains unknown. The maintenance of neuronal polarity depends on the AIS region in which proteins are stabilized and organized by ankyrin G (ankG). In the AIS, MTs are stabilized by the binding of the MT plus-end-binding protein 3 (EB3) to ankG ${ }^{21}$, and HDAC6, as a MT-capping protein, may contribute to MT stability ${ }^{22}$. It is thus important to establish the possible link between the increased acetylation found in $\mathrm{AD}$, the activity of HDAC6 and A $\beta$.

Here we provide evidence that the contact of extracellular $A \beta$ with the plasma membrane is sufficient to induce growth cone collapse by activation of the RhoA/ROCK-dependent pathway and increase in actin retrograde flow in neuronal growth cones, with destructive effect on neurite outgrowth. In addition, we show for the first time that internalized A $\beta$ inhibits HDAC6 inducing an increase in the acetylation of $\alpha / \beta$-tubulin heterodimers and of tau. Furthermore, we demonstrate that $A \beta$ increased MT dynamics in the AIS, disrupting the barrier and leading to missorting of tau in minor neurites, effects that are prevented by HDAC6 overexpression. We propose that activation of the RhoA-ROCK pathway by extracellular $A \beta$ and inhibition of HDAC6 by intracellular $A \beta$ result in cytoskeletal instability in neurites and in the AIS, leading to loss of neuronal polarity.

\section{Results}

Extracellular A $\beta$ induces neurite retraction. Neurite outgrowth and retraction are processes tightly regulated by cytoskeletal dynamics. Previous studies reported that $A \beta$ fibrils reduce neurite outgrowth via activation of RhoA in a neuroblastoma cell line ${ }^{23}$ and in hippocampal neurons ${ }^{24}$. $A \beta$ is released extracellularly on cleavage of amyloid precursor protein at the plasma membrane or in endosomes, and the monomeric form is present in the extracellular milieu before aggregation might take place. To investigate whether $A \beta$ is able to influence axonal elongation, we used the purified soluble form of $A \beta$. Its molecular state was assessed by western blot and atomic force microscopy (AFM) (Supplementary Fig. 1a,b) showing that $\mathrm{A} \beta$ was mostly in the monomeric form and few oligomers were present after 1-h incubation in neuronal medium. Embryonic hippocampal neurons at 3 days in vitro (DIV) were incubated with $1 \mu \mathrm{M} \mathrm{A} \beta$ for $1 \mathrm{~h}$ and the length of axons was measured by axonal tracing. In the presence of $A \beta$, the average axonal length was decreased by $31.7 \%$ of control axons (Fig. 1a). The longest neurite was considered as axon, as demonstrated by the polarized localization of tau (Fig. 1b). The average growth of 3 DIV hippocampal neurons in our conditions was of $\sim 20 \mu \mathrm{m} \mathrm{h}^{-1}$. The length of the axon in neurons incubated with $A \beta$ for $1 \mathrm{~h}$ was $\sim 35 \mu \mathrm{m}$ shorter with respect to that of control neurons and was therefore suggestive of axonal retraction. To better understand the mechanism underlying retraction of the specific neurite under the influence of extracellular $A \beta$, we used optically driven $A \beta$ - or scrambled $A \beta$-coated microspheres brought in contact with the neck of axonal growth cone (Fig. 1c). Coating of microspheres was verified by confocal imaging (Fig. 1d), and the local application of $A \beta$ bound to microspheres induced growth cone collapse, as indicated by a $52.9 \%$ reduction in the growth cone surface area after $30 \mathrm{~min}$ (Fig. 1e), with complete collapse after $40 \mathrm{~min}$. The effect of $A \beta$ was prevented by the inhibitor of Rho-associated kinase (ROCK) Y27632, and by the myosin II inhibitor, blebbistatin, indicating the involvement of ROCK-dependent pathway and actin retrograde flow in the collapse of the growth cone (Fig. 1c,e).

Extracellular A $\beta$ alters growth cone adhesion and motility. To monitor cytoskeletal dynamics in the axonal growth cone throughout the external local application of $A \beta$-coated microspheres, we performed simultaneous bright-field imaging of the neuron and force spectroscopy measurement on the adhered coated microsphere positioned on the neck of the growth cone (Supplementary Fig. 2a). The movement of the microsphere, measured through a force-clamp system ${ }^{25}$, is dissected in two distinct components (Supplementary Fig. 2b and Methods section). The low-frequency component of the motion is associated with the cytoskeletal dynamics underlying the remodelling of the membrane-leading edge, and it is recorded through the PIEZO stage, which tracks the bead position (Supplementary Fig. 2c, upper panel). The high-frequency component of the motion, representing the 


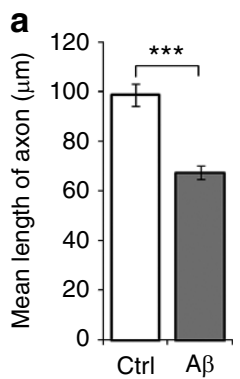

b

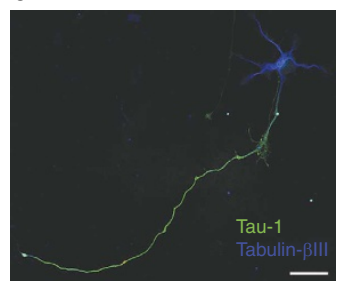

d

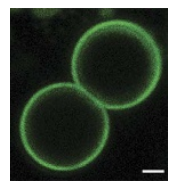

e

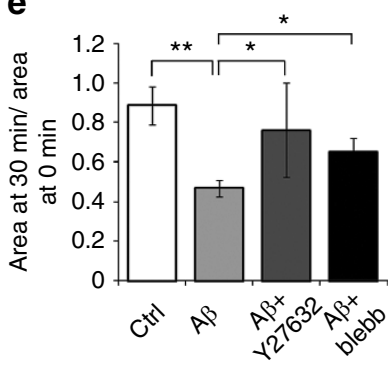

C

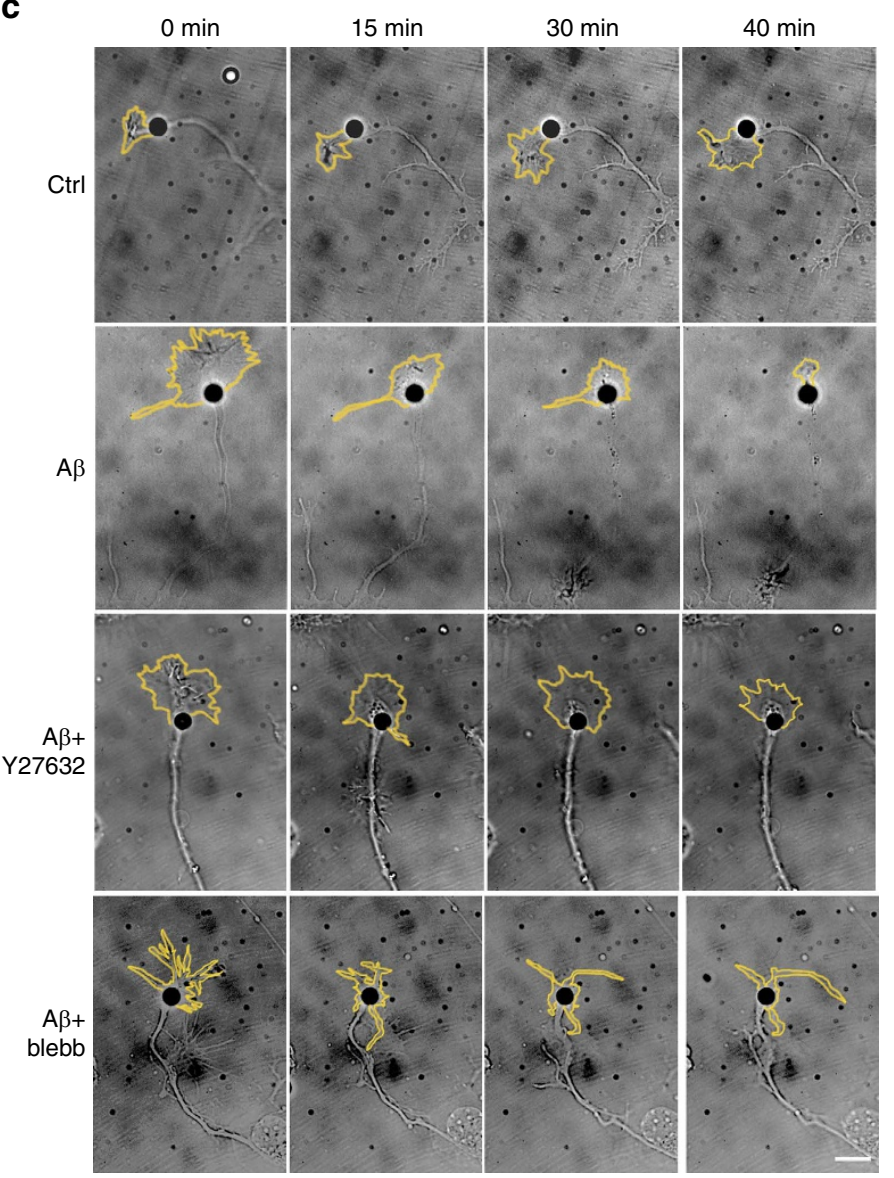

Figure 1 | Extracellular A $\boldsymbol{\beta}$ induces neurite retraction via ROCK-dependent pathway. (a) Bar graph showing the mean axonal length of neurons incubated either with $0.05 \%$ DMSO (ctrl, 98.79 $\pm 4.44, n=134$ ) or with $1 \mu \mathrm{M} \mathrm{A} \beta(A \beta, 67.51 \pm 2.77, n=159)$ for $1 \mathrm{~h}$, measured by axon tracing. Statistical significance determined by two-tailed $t$-test, ${ }^{\star \star} P<0.001$. (b) Fluorescence analysis of tau (green) and tubulin- $\beta$ III (blue) localization in cultured hippocampal neuron at 3 DIV, showing tau polarization in axons. Scale bar, $25 \mu \mathrm{m}$. (c) Images taken at 0, 15, 30 and 40 min from time-lapse movies of embryonic hippocampal neurons in the presence of optically driven microspheres coated with scrambled $A \beta$ (top panels), $A \beta$ (middle panels), with $A \beta$ and $10 \mu \mathrm{M}$ Y27632, a ROCK inhibitor (lower panels), or with $A \beta$ and $10 \mu \mathrm{M}$ blebbistatin, a myosin II inhibitor. The growth cone area (highlighted in yellow) shows a reduction upon contact with the $A \beta$-coated microsphere that is prevented by $Y 27632$ and by blebbistatin. Scale bar, $25 \mu \mathrm{m}$. (d) Confocal image showing successful coating of microspheres with fluorescently labelled $A \beta$ peptide. Scale bar, $1 \mu \mathrm{m}$. (e) Bar graph showing the ratio between the growth cone area at the end and that at the start of time-lapse movies as in c. Scrambled $A \beta$ (ctrl, 1.05 $\pm 0.11, n=6), A \beta(A \beta, 0.43 \pm 0.04, n=7), A \beta+10 \mu M$ $Y 27632(A \beta+Y 27632,0.93 \pm 0.24, n=4)$ and $A \beta+10 \mu M$ blebbistatin $(A \beta+$ blebb, $0.70 \pm 0.07, n=3)$. Statistical significance determined by two-tailed t-test, ${ }^{\star \star} P<0.001(\mathrm{ctrl} / \mathrm{A} \beta),{ }^{\star} P=0.034(\mathrm{~A} \beta / \mathrm{A} \beta+\mathrm{Y} 27632)$, ${ }^{\star} P=0.009(\mathrm{~A} \beta / \mathrm{A} \beta+$ blebb). In $\mathbf{a}$ and $\mathbf{e}$, data are expressed as mean \pm s.e.m.

Brownian fluctuation of the bead, is associated with the strength of the adhesion between the bead and the cell membrane, and is measured by the quadrant photodiode (QPD) (Supplementary Fig. 2c, lower panel). The variance of the QPD signal decreases when the adhesion contact is stabilized through the binding to a receptor, or by receptors clustering at the bead contact. The lowand high-frequency components of the bead motion are prevalently influenced by actin polymerization ${ }^{26,27}$ and myosin II motor contraction ${ }^{28}$.

The change in the variance associated with the presence of the bead could be biased on the presence of previously established focal adhesions at the contact point that strongly influence the local cytoskeletal organization. In our experimental conditions, the bead was attached to the apical surface, where two-dimensional cultured cells are normally unable to establish adhesion structures, due to the lack of mechanical constraint. Therefore, the bead positioned on the surface of the growth cone can be considered the first and only mechanical constraint experienced by the cell. Correspondingly, the observed dynamics are associated with the formation of an adhesion contact caught at its onset, in a reproducible way between all neurons examined. Application of $A \beta$-coated microspheres induced a large and fast decrease in the variance with respect to control that was prevented by cell treatment with Y27632 or blebbistatin (Supplementary Fig. 2d; Fig. 2a), suggesting that in the presence of $A \beta$, the tightening of the adhesion with the membrane is faster and stronger, and involves activation of ROCK. To quantify A $\beta$-induced growth cone collapse (Fig. 1e), we monitored the 

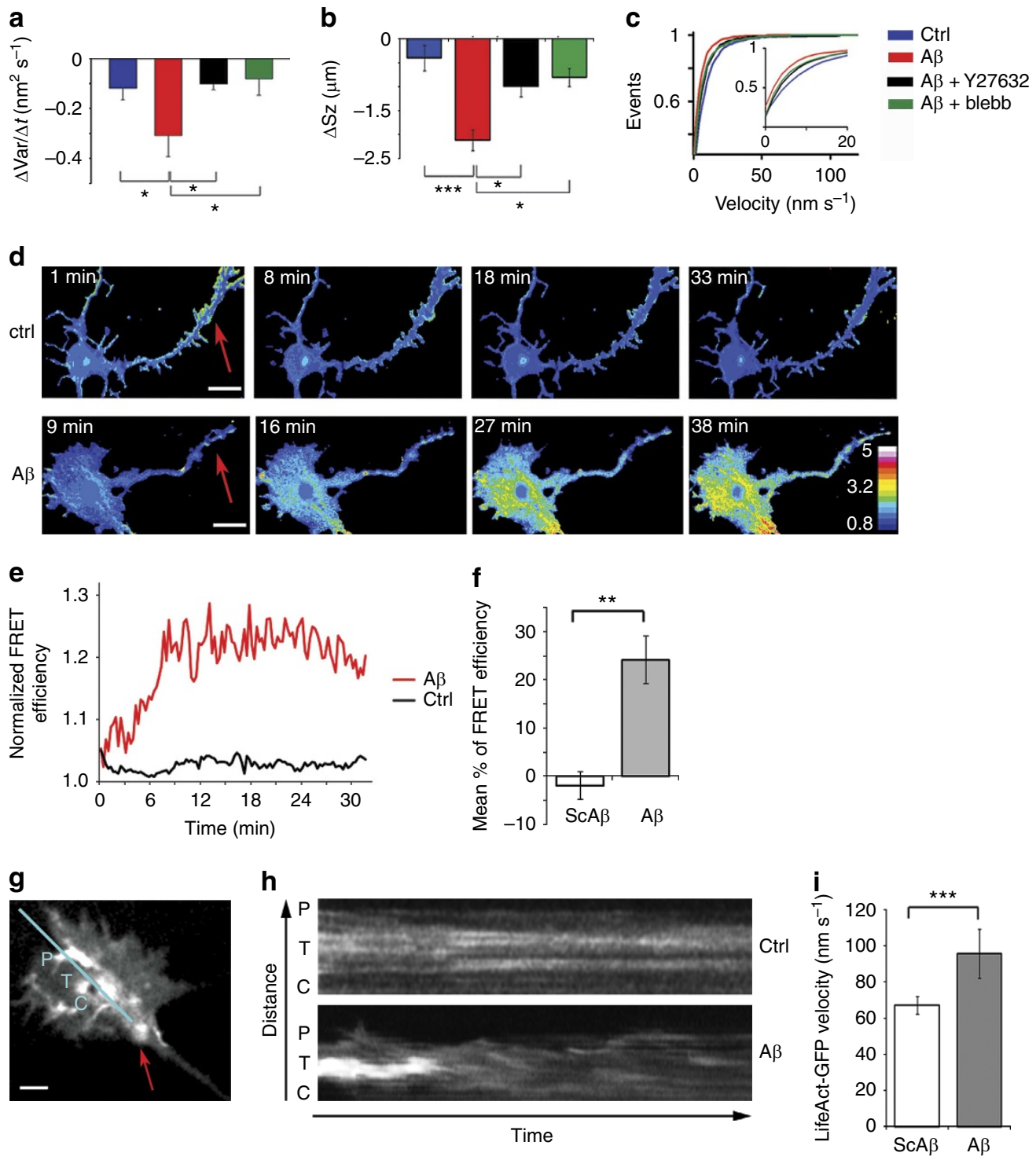

Figure 2 | Extracellular $\mathbf{A} \boldsymbol{\beta}$ alters cytoskeleton dynamics by activating RhoA and increases F-actin retrograde flow. (a) Quantification of the Brownian motion variance decrease. Scrambled $A \beta$ (blue, $-0.15 \pm 0.05, n=8$ ); $A \beta$ (red, $-0.31 \pm 0.08, n=9$ ); $A \beta+$ blebb (green, $-0.08 \pm 0.06, n=5$ ); and $A \beta+Y 27632$ (black, $-0.1 \pm 0.02, n=5)$. Statistical significance determined by one-way ANOVA, ${ }^{\star} P=0.034(\operatorname{ctrl} / A \beta),{ }^{\star} P=0.035(A \beta / A \beta-Y 27632)$, ${ }^{\star} P=0.033$ (A $\beta$ /A $\beta$-blebb). (b) Quantification of the decrease in the $z$ coordinate. Scrambled $A \beta$ (blue, $-0.4 \pm 0.26$ ); $A \beta($ red, $-2.11 \pm 0.21$ ); $A \beta+$ blebb (green, $-0.8 \pm 0.18$ ); and $A \beta+Y 27632$ (black, $-1.1 \pm 0.19$ ). $n$ as in a. Statistical significance determined by one-way $A N O V A, \star \star \star P<0.001$ (ctrl/A $\beta$ ), ${ }^{\star} P=0.028(A \beta / A \beta+Y 27632),{ }^{\star} P=0.012(A \beta / A \beta+$ blebb). (c) Normalized cumulative histograms of the microsphere velocity. An enlargement is shown in the inset. Statistical significance determined by two sample Kolmogorov-Smirnov test, $P<10^{-26}$ (ctrl/A $\beta$ ), $P=0.002(A \beta / A \beta+b l e b b), P<10^{-8}$ $(A \beta / A \beta+Y 27632), P=0.002$ (ctrl/A $\beta+Y 27632), P<10^{-28}$ (ctrl/A $\beta+$ blebb), $P=0.04$ (A $\beta+$ blebb/A $\left.\beta+Y 27632\right)$. (d) Images of YFP/CFP ratio in neurons overexpressing the FRET probe Raichu-RhoA. A scrambled A $\beta$ (top panels) or $A \beta$ (bottom panels)-coated microsphere was attached to the neurite (red arrows). A pseudocoloured scale of the YFP/CFP ratio is shown in the lower right image. Scale bars: upper row, $12 \mu \mathrm{m}$; lower row, $10 \mu \mathrm{m}$. (e) Representative curves of the normalized FRET efficiency in neurons overexpressing Raichu-RhoA and treated as in d. Scrambled $A \beta$ (black) and $A \beta$ (red). (f) Quantification of FRET efficiency in the neurite of neurons treated as in d. Scrambled $A \beta$ (ctrl, $-1.93 \pm 2.85, n=6)$ and $A \beta(A \beta, 24.21 \pm 4.93$, $n=10$ ). Statistical significance determined by two-tailed $t$-test, ${ }^{\star \star} P=0.0018$. (g) Example of a neuron electroporated with LifeAct-GFP and put in contact with a microsphere (red arrow). Kymographs were constructed along the light blue line. Scale bar, $5 \mu$ m. (h) Representative kymographs obtained from time-lapse movies of LifeAct-GFP-electroporated neurons in the presence of scrambled A $\beta$ - (ctrl, top panel) or A $\beta$-coated microspheres (A $\beta$, bottom panel). In $\mathbf{g}$ and $\mathbf{h}$, peripheral domain (P), transition zone ( $T$ ) and central domain (C). (i) Quantification of LifeAct-GFP velocity obtained from kymographs as in $\mathbf{h}$. Scrambled $A \beta(S c A \beta, 67.70 \pm 3.91$, slopes $n=54)$ and $A \beta(A \beta, 92.95 \pm 6.51$, slopes $n=49)$. Statistical significance determined by two-tailed $t$-test, ${ }^{\star \star \star} P=0.001$. In $\mathbf{a}, \mathbf{b}, \mathbf{f}$ and $\mathbf{i}$, data are expressed as mean \pm s.e.m. ANOVA, analysis of variance.

vertical position $\mathrm{Sz}$ of the bead as a function of time (Supplementary Fig. 2e). The bead was attached at the neck of the growth cone, and, with $\mathrm{A} \beta$-coated microspheres, the height of the bead decreased by $\sim 2 \mu \mathrm{m}$ in $45 \mathrm{~min}$, due to emptying of the growth cone content that subsequently leads to axon shrinking, while with scrambled $A \beta$-coated microsphere, we observed fluctuation of the bead height in the range of few hundred nanometres, not associated with decrease in thickness of the growth cone neck. The shrinking effect induced by $A \beta$-coated microspheres was prevented by incubation of neurons with Y27632 or blebbistatin (Fig. 2b). Similar difference between A $\beta$ and control appeared when we looked at the average microsphere 
velocity, an index of active reshaping of the cell associated with cytoskeleton dynamics (Supplementary Fig. 2f). The normalized cumulative histogram of the microsphere velocity (Fig. 2c) shows that with $A \beta$-coated microspheres (in red) the curve is clearly shifted towards zero, indicating impaired assembly and disassembly of cytoskeletal filaments. The treatment with Y27632 or blebbistatin partially prevented the inhibition of cell reshaping due to $A \beta$.

Taken together, these results suggest that axon retraction is induced by increased myosin II-mediated actin retrograde flow, with ensuing substantial and fast stabilization of cell-microsphere adhesion contact. Furthermore, the similarity in the velocity distribution obtained with Y27632 or blebbistatin suggests that ROCK and myosin II are part of the same pathway affected by local delivery of $A \beta$ on the neuronal surface.

Extracellular $\mathbf{A} \beta$ increases actin retrograde flow. The involvement of ROCK and myosin II in A $\beta$-driven growth cone collapse was suggestive of the activation of RhoA, whose downstream target is ROCK. We took advantage of the intramolecular fluorescence-resonance energy transfer (FRET) probe Raichu, a biosensor of RhoA activity ${ }^{29}$, to follow the localized activation of RhoA in the presence of $A \beta$-coated microspheres, optically driven in contact with the distal part of the axon of cultured neurons (Fig. 2d). Images in the YFP and CFP emission wavelengths were acquired separately for $30 \mathrm{~min}$ and FRET efficiency was calculated as acceptor/donor ratio (Fig. 2e). The contact with $A \beta$-coated microsphere induced a $24 \%$ increase in FRET efficiency compared with that in control neurons (in contact with scrambled $A \beta$-coated microsphere) (Fig. 2f).

To confirm the activation of RhoA, which is known to regulate actin retrograde flow, actomyosin contraction was evaluated in the presence of $A \beta$-coated microspheres. Neurons were electroporated with LifeAct-green fluorescent protein (GFP), to follow the movement of microfilaments, to which LifeAct specifically binds. Kymographs were assembled from live-imaging movies of the growth cone (Fig. $2 \mathrm{~g}$ ) to measure the rate of actin retrograde flow in the presence of $A \beta$-coated or scrambled $\mathrm{A} \beta$-coated microspheres (Fig. 2h). Statistical analysis demonstrated a significant $\sim 40 \%$ increase of actin retrograde flow induced by extracellular $A \beta$ compared with scrambled $A \beta$ (Fig. 2i), suggesting that actin retrograde flow might be responsible for the observed growth cone collapse in neurons exposed to $A \beta$.

A $\beta$ increases MT dynamics and increases acetylated tubulin. RhoA activation has been previously shown to stabilize $\mathrm{MT}^{30}$, which show long half-life and result capped at their plus end. Post-translational modifications of MT, such as acetylation and detyrosination, or tyrosination have been studied as markers for stabilized MT or for dynamic MT, respectively ${ }^{31}$. We therefore analysed the levels of acetylated tubulin (ac-tub) by immunofluorescent staining of neurons exposed or not to $A \beta$. An increase in ac-tub was evident after $1 \mathrm{~h}$ of exposure to $A \beta$ and quantified as $\sim 30 \%$ higher than in control, while tubulin$\beta$ III showed no change. Taxol, a MT stabilizer, and tubacin, a specific inhibitor of HDAC6 deacetylase, were used as positive controls and induced an increase of $\sim 50$ and $\sim 40 \%$, respectively, of the signal of ac-tub (Fig. 3a,b). RhoA activation by $A \beta$ and stabilization of MT would also result in inhibition of MT dynamics ${ }^{32}$. To determine MT dynamics, we analysed the rate of movement of the fluorescent comets of $\mathrm{EB} 3$, in neurons overexpressing EB3-GFP on incubation with soluble A $\beta$ by total internal reflection live-imaging microscopy. Surprisingly, we observed that $A \beta$ was able to increase the average speed of
EB3-GFP comets by $\sim 40 \%$ (Fig. 3c), in apparent contradiction with the effect of $A \beta$ on acetylation of MT; however, also in the presence of tubacin, which induced MT acetylation, the speed of EB3 comets was almost doubled. The analysis of A $\beta$ effect on MT was performed after 1-h incubation with the peptide, to allow internalization of $A \beta$ (Supplementary Fig. 3); therefore, the effects of $A \beta$ will result from its action both at the cell membrane and in the intracellular space.

A $\boldsymbol{\beta}$ inhibits HDAC6. We therefore wondered whether the increase in acetylation could be due to ac-tub hetereodimers rather than to acetylated MT, as MT were in a more dynamic state in the presence of $A \beta$, as shown by the measure of EB3 velocity. To address this hypothesis, we performed ac-tub immunostaining after permeabilization of neurons with Tx-100, to induce leakage of heterodimers, keeping only polymerized MT inside the cell. Indeed, in neurons treated with $1 \mu \mathrm{M}$ taxol and permeabilized, the signal of ac-tub was stronger than that of control, while after $1 \mathrm{~h}$ of exposure to $A \beta$, and similarly after exposure to tubacin, the signal of ac-tub was comparable to that of control neurons (Fig. 4a,b). Physiologically, acetylation of tubulin occurs preferentially on polymerized $\mathrm{MT}^{33}$, whereas deacetylation of $\alpha$-tubulin by HDAC6 is thought to occur when $\alpha$-tubulin is in a heterodimeric state ${ }^{34}$. Therefore, HDAC6 might be a target of $A \beta$, translating its effect on tubulin acetylation. To measure the catalytic activity of HDAC6, we performed an in vitro assay using purified HDAC6 together with $A \beta$ or the HDAC inhibitor trichostatin A (TSA) as a positive control. Statistical analysis indicated $\sim 25 \%$ and $\sim 12 \%$ significant inhibition of HDAC6 activity by $1 \mu \mathrm{M}$ and $100 \mathrm{nM} \mathrm{A \beta}$, respectively, with TSA inducing a $\sim 50 \%$ decrease in respect to control (Fig. 4c), suggesting direct inhibitory interaction of $A \beta$ with HDAC6, at least in vitro. The increase in ac-tub induced by $\mathrm{A} \beta$ was confirmed by western blot analysis. Lysates of neurons treated with $A \beta$ show increase in ac-tub as expected (Fig. $4 d$, ac-tub, first two lanes). Analysis of lysates from neurons fixed after the incubation period, to maintain MT and monomers in the original conditions of polymerization, confirmed that exposure of neurons to $A \beta$ induced an increase of ac-tub besides MT, appreciable as a band at $52 \mathrm{kDa}$ and bands at higher molecular weight, respectively, similarly to the effect of exposure to tubacin. Taxol, consistently with the results shown in the immunofluorescence experiment on permeabilized neurons, preferentially induces acetylation of MT (Fig. 4d, ac-tub, last four lanes; Fig. 4e). The effect of internalized $A \beta$, arising at later time points in respect of the one induced by contact with the plasma membrane, appears to be associated with increased MT dynamics. To further examine the action of $A \beta$ on MT, we assessed the number of catastrophes/pauses, finding a significant $\sim 50 \%$ increase on incubation with $\mathrm{A} \beta$ (Fig. $4 \mathrm{f}$ ), possibly because of the combined action of $A \beta$ on MT and actin retrograde flow, suggesting that MTs are in a highly unstable state after $A \beta$ treatment.

$\mathrm{A} \beta$ increases acetylated tau and induces missorting of tau. Destabilization of MT in AD pathology is mainly attributed to the dissociation of tau, the main axonal microtubule-associated protein (MAP). In the light of a recent study showing that HDAC6, in addition to SIRT1 (ref. 17), also deacetylates tau ${ }^{16}$, we analysed the level of ac-tau on $\mathrm{A} \beta$ treatment. After exposure of neurons to $100 \mathrm{nM}$, or $1 \mu \mathrm{M} A \beta$ or $100 \mathrm{nM}$ TSA, tau immunoprecipitated from neuronal lysates showed increased acetylation (Fig. 5a,b). As acetylation of tau has been shown to correlate with decreased MT-binding capability ${ }^{16}$, which induces disruption of tau retrograde barrier ${ }^{15}$, we analysed tau 
a

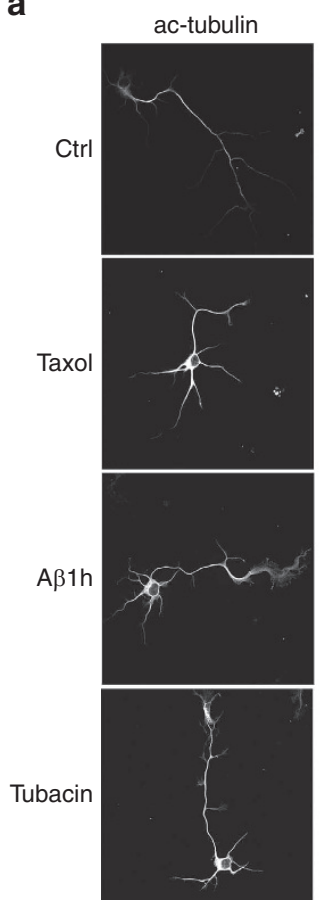

Tubulin- $\beta$ III

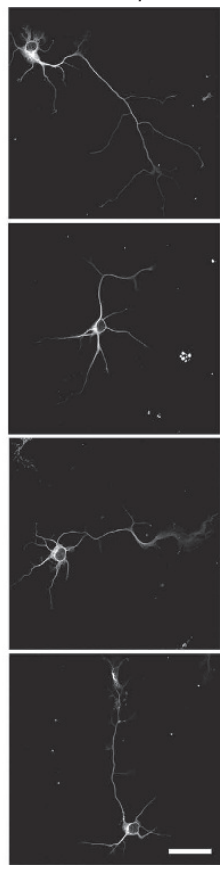

b
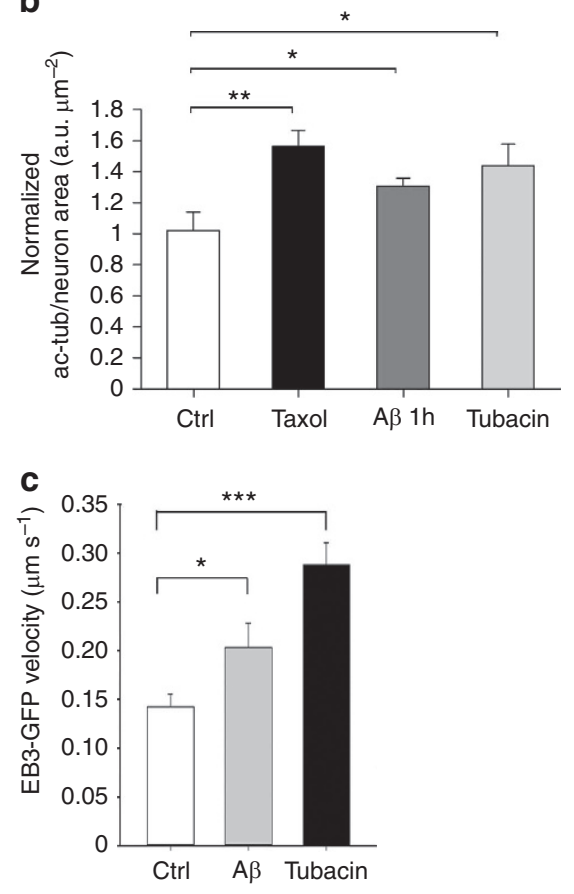

Figure 3 | A $\boldsymbol{\beta}$ increases the MT plus-end dynamics and acetylated tubulin. (a) Fluorescence analysis of acetylated tubulin (ac-tub) in hippocampal neurons treated with either $0.05 \%$ DMSO (ctrl) for $1 \mathrm{~h}, 1 \mu \mathrm{M}$ taxol for $30 \mathrm{~min}, 100 \mathrm{nM} \mathrm{A} \beta$ for $1 \mathrm{~h}$ or $10 \mu \mathrm{M}$ tubacin for $1 \mathrm{~h}$ showing increased intensity of ac-tub staining in A $\beta$-, tubacin- and taxol-treated neurons compared with control. Scale bar, $25 \mu \mathrm{m}$. (b) Quantification of ac-tub staining intensity normalized to the neuron area calculated from the area of tubulin- $\beta$ III immunofluorescence. Hippocampal neurons were treated with either $0.05 \%$ DMSO for $1 \mathrm{~h}(\mathrm{ctrl}, 1 \pm 0.12, n=24), 1 \mu \mathrm{M}$ taxol for $30 \mathrm{~min}$ (taxol, $1.56 \pm 0.10, n=30), 100 \mathrm{nM} \mathrm{A} \beta$ for $1 \mathrm{~h}(\mathrm{~A} \beta 1 \mathrm{~h}, 1.31 \pm 0.05, n=31$ ) or $10 \mu \mathrm{M}$ tubacin for $1 \mathrm{~h}$ (tubacin, $1.44 \pm 0.14, n=23$ ). Statistical significance determined by one-way $A N O V A,{ }^{\star \star} P=0.002$ (ctrl versus taxol), ${ }^{\star} P=0.04$ (ctrl versus $A \beta$ ), ${ }^{\star} P=0.02$ (ctrl versus tubacin). (c) Bar graph showing the mean speed of GFP comets in EB3-GFP-electroporated neurons, preincubated with $0.05 \%$ DMSO (ctrl, $0.14 \pm 0.01, n=22), 100 \mathrm{nM} \mathrm{A} \beta$ for $1 \mathrm{~h}(A \beta, 0.20 \pm 0.02, n=16)$ or $10 \mu \mathrm{M}$ tubacin for $1 \mathrm{~h}(0.29 \pm 0.02, n=6)$, and imaged for $90 \mathrm{~s}$. Statistical significance determined by one-way ANOVA, ${ }^{\star} P=0.039,{ }^{\star \star \star}{ }^{\star \star} P<0.001$. In $\mathbf{b}$ and $\mathbf{c}$, data are expressed as mean \pm s.e.m. ANOVA, analysis of variance.

localization on exposure to $\mathrm{A} \beta$. In $3 \mathrm{DIV}$ neurons treated with $100 \mathrm{nM} \mathrm{A} \beta$, tau-1 staining was present also in minor neurites (Fig. 5c) and the percentage of neurons with tau-1 localization restricted to the axon was significantly decreased, being $\sim 2.5$ fold less than that of control; inhibition of HDAC6 by tubacin yielded similar results (Fig. 5d). Tau missorting was observed both in young neurons at 3 DIV and in neurons at later stage of development (14 DIV) (Fig. 5c; Supplementary Fig. 4a). Neurons were co-electroporated with HDAC6-FLAG together with GFP, obtaining a transfection efficiency of $15-20 \%$, and GFP-expressing neurons were chosen to analyse tau localization. Importantly, overexpression of HDAC6 prevented A $\beta$-driven missorting of tau, which was polarized in the axon twofold more than in $A \beta$-treated neurons (Fig. $5 c, d$ ). Taken together, these results indicate that the inhibition of HDAC6 induced by $A \beta$ is sufficient to increase acetylation of tau and to induce tau missorting in minor neurites, and that restoring of HDAC6 function might counteract $\mathrm{A} \beta$ phenotype.

A $\beta$ disrupts the integrity of the AIS. In the maintenance of tau retrograde barrier, not only tau binding to $\mathrm{MT}$ is fundamental but also MT stability per se in the region overlapping with AIS $^{15}$ where EB3 is found enriched and less mobile ${ }^{21}$. To understand the possible mechanism of action of $A \beta$ on axonal polarity in view of the observed missorting of tau-1, we measured the rate of movement of EB3 in the AIS (Fig. 6a). Kymographs of the EB3GFP comets in the AIS regions of control neurons showed immobile EB3, whereas in A $\beta$-treated, as in tubacin-treated neurons, EB3 appeared more mobile (Fig. 6b). The speed of EB3 comets in the AIS of $A \beta$ - and tubacin-treated neurons was increased by $45.3 \%$ and by $33.1 \%$, respectively, in comparison with that of control (Fig. 6c; Supplementary Movies 1-3). Importantly, the destabilizing effect of $A \beta$ on the MT in the AIS was no longer observed in neurons overexpressing HDAC6 (Fig. 6b,c; Supplementary Movies 4-5). As HDAC6 had been implicated in the maintenance of AIS $^{20}$ by its MT-binding activity, we investigated whether $A \beta$ could modify the integrity of AIS by the analysis of ankG localization. Neurons exposed to A $\beta$ and tubacin showed ankG staining no longer restricted to the AIS as in control neurons, but dispersed along the length of the axon (Fig. 6d). The length of ankG staining along the axon was increased by $\sim 30 \%$ and $\sim 40 \%$ in $A \beta$ - and tubacin-treated neurons, respectively (Fig. 6e). AnkG dispersion was appreciable both in young neurons at $4 \mathrm{DIV}$ and in neurons at later stage of development (14 DIV) (Fig. 6d; Supplementary Fig. 4b). Given that previous studies provided direct evidence for a physiological role of ankG in the clustering of voltage-gated sodium channels into the axon initial segment ${ }^{35}$, we investigated whether $A \beta$ treatment could interfere with the ability of the neuron to fire action potentials upon injection of current at the soma in whole-cell patch-clamp recordings. Examination of action potential profile revealed a marked increase of the spike threshold (defined by the membrane potential at which $\mathrm{d} V / \mathrm{d} t$ of the spike crossed $15-20 \mathrm{mVms}^{-1}$ ) for $\mathrm{A} \beta$-treated neurons compared with the control ones (Fig. 6f-h). Taken together, these results indicate that disruption of the AIS barrier by $A \beta$ results in impaired action potential firing. 
a

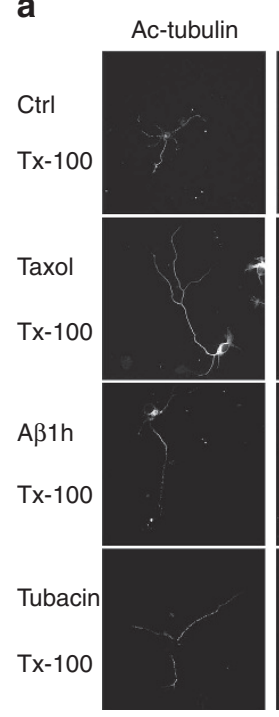

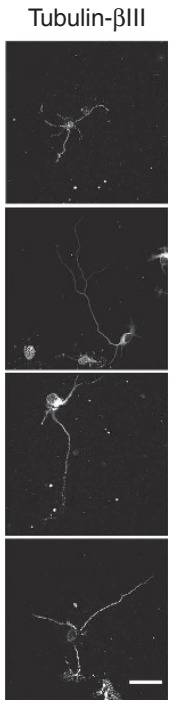

d b

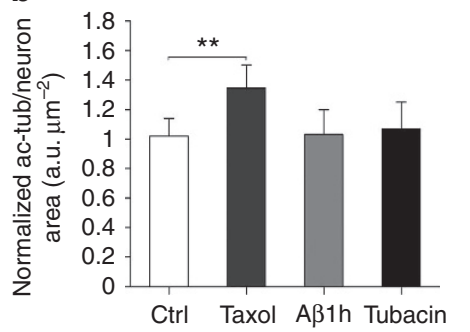

Ctrl Taxol Aß1h Tubacin c

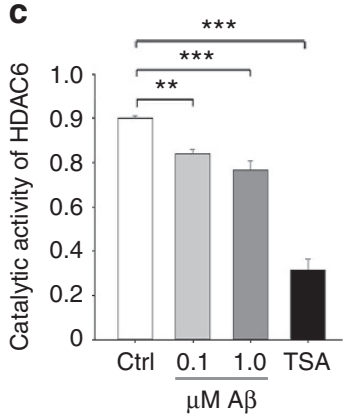

f

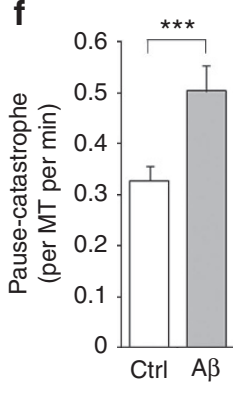

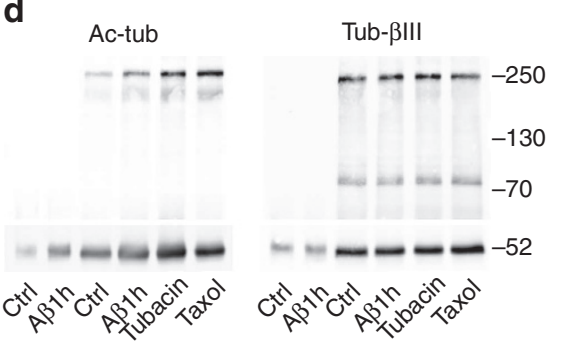
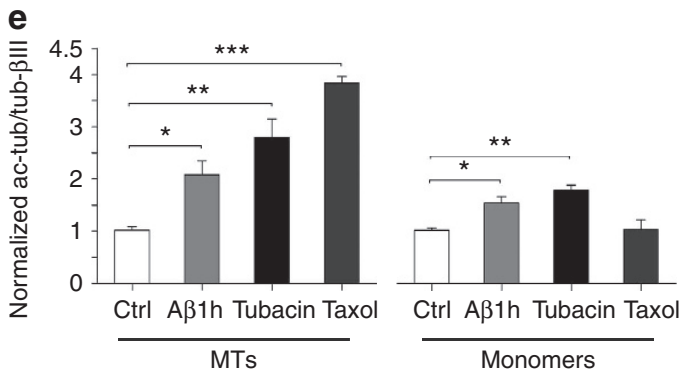

Figure 4 | A $\beta$ inhibits HDAC6 and increases acetylation of tubulin heterodimers. (a) Fluorescence analysis of ac-tub in neurons treated with either $0.05 \%$ DMSO (ctrl) for $1 \mathrm{~h}, 1 \mu \mathrm{M}$ taxol for $30 \mathrm{~min}, 100 \mathrm{nM} \mathrm{A} \beta$ for $1 \mathrm{~h}$ or $10 \mu \mathrm{M}$ tubacin for $1 \mathrm{~h}$, and permeabilized. Scale bar, $25 \mu \mathrm{m}$. (b) Quantification of ac-tub staining intensity normalized to neuronal area calculated from tubulin- $\beta \| I$ immunofluorescence in permeabilized neurons. Neurons were treated with either $0.05 \%$ DMSO for $1 \mathrm{~h}$ (ctrl, $1 \pm 0.12, n=25), 1 \mu \mathrm{M}$ taxol for $30 \min (1.35 \pm 0.15, n=26), 100 \mathrm{nM} \mathrm{A \beta}$ for $1 \mathrm{~h}(\mathrm{~A} \beta, 1.03 \pm 0.17, n=30)$ or $10 \mu \mathrm{M}$ tubacin for $1 \mathrm{~h}$ (tubacin, $1.07 \pm 0.18, n=16$ ). Statistical significance determined by one-way ANOVA, ${ }^{\star \star} P=0.035$. (c) In vitro assay of the catalytic activity of HDAC6 in the presence of either DMSO (ctrl, $1.00 \pm 0.00, n=10), 100 n M A \beta(A \beta 0.1,0.86 \pm 0.02, n=8), 1 \mu M A \beta(A \beta 1.0,0.78 \pm 0.04, n=8)$ or $50 \mathrm{nM}$ trichostatin $\mathrm{A}(\mathrm{TSA}, 0.14 \pm 0.05, n=10)$ normalized to the control. Statistical significance determined by one-way ANOVA, ${ }^{\star \star} P=0.006$, ${ }^{\star \star \star} P<0.001$. (d) Representative immunoblot showing ac-tub and tubulin- $\beta$ III in MT (higher bands) and monomers (band at $52 \mathrm{kDa}$ ) from lysate of not fixed (first two lanes) or fixed (last four lanes) neurons treated with $0.05 \%$ DMSO for $1 \mathrm{~h}$ (ctrl), $100 \mathrm{nM} \mathrm{A \beta}$ for $1 \mathrm{~h}$ ( $\mathrm{A} \beta 1 \mathrm{~h}$ ), $10 \mu \mathrm{M}$ tubacin for $1 \mathrm{~h}$ (tubacin) or $1 \mu \mathrm{M}$ taxol for $30 \mathrm{~min}$ (taxol). One out of ten of lysate from non-fixed samples was loaded. (e) Quantification of the ratio between ac-tub and tubulin- $\beta$ III levels performed on the sum of the bands above $52 \mathrm{kDa}(\mathrm{MTs})$ and on the band at $52 \mathrm{kDa}$ (monomers) from neurons treated as in a and fixed, $n=3$. Statistical significance determined by one-way ANOVA. MT: ${ }^{\star} P=0.04$ (ctrl/A $\beta 1 \mathrm{~h}, 2.08 \pm 0.27$ ); ${ }^{\star \star} P=0.003$ (ctrl/tubacin, $2.80 \pm 0.35$ ); ${ }^{\star \star \star} P<0.001$ (ctrl/taxol, $3.84 \pm 0.12$ ). Monomers: ${ }^{\star} P=0.04$ (ctrl/A 1 h, $\left.1.53 \pm 0.12\right) ;{ }^{\star \star} P=0.004$ (ctrl/tubacin, $\left.1.77 \pm 0.10\right) ;(\mathrm{ctrl} / \mathrm{taxol}, 1.02 \pm 0.18)$. (f) Bar graph showing the number of pause/catastrophe in neurons electroporated as in Fig. $3 \mathrm{c}$ and incubated with $0.05 \%$ DMSO (ctrl, $0.33 \pm 0.03, n=401 \mathrm{from} 43$ neurons) or $100 \mathrm{nM} \mathrm{A \beta}\left(\mathrm{A} \beta, 0.50 \pm 0.05, n=222\right.$ from 28 neurons). Statistical significance determined by two-tailed $t$-test, ${ }^{\star \star \star} p<0.001$. In $\mathbf{b}, \mathbf{c}, \mathbf{e}$ and $\mathbf{f}$, data are expressed as mean \pm s.e.m. ANOVA, analysis of variance.

\section{Discussion}

In this study, we provide evidence that submicromolar concentration of $A \beta$ is able to alter actin and MT dynamics, via RhoA activation and inhibition of HDAC6, respectively. The altered cytoskeletal dynamics led to growth cone collapse, defect in axon elongation, and disruption of MT stability in the AIS, ultimately interfering with the maintenance of neuronal polarity.

Using optical tweezers and microspheres coated with $A \beta$, we were able to study the local effect of extracellular $A \beta$, which induces growth cone collapse and neurite retraction in developing neurons. Our data suggest that soluble extracellular $A \beta$ is sufficient in activating RhoA/ROCK pathway ${ }^{36}$, and also increases actin retrograde flow in a ROCK/myosin II-dependent manner. Indeed, inhibition of ROCK activity counteracted the effect of extracellular $A \beta$, preventing growth cone collapse and suggesting the possibility of a protective role of ROCK inhibitors in $\mathrm{AD}$. The initial functional characterization of the members of the Ras superfamily of GTPases established actin cytoskeleton as their main $\operatorname{target}^{37}$, and later those observations were extended to reveal their function in the regulation of MT organization ${ }^{38}$. Growth cone movement is necessary for elongation and pathfinding of developing neurons. In ageing brain and in $\mathrm{AD}$, an impairment of adult neurogenesis was found ${ }^{39}$ that deepen the symptoms of neurodegenerative diseases due to loss of neurons. Therefore, an alteration in developmental processes that are fundamental for the correct migration of neurons might be at the basis of cell death in the areas of adult neurogenesis. These processes depend on coordinated action of microfilaments and $M^{40}$, prompting further investigation on the effect of $A \beta$ on actin-MT crosstalk.

To analyse MT dynamics, $A \beta$ was added to the media of neurons, in which case the combined effect of $A \beta$ both from the extracellular milieu and from inside the cells on its internalization was observed. We found that $A \beta$ affected MT dynamics by inhibiting HDAC6, a deacetylase of tubulin and tau. While $A \beta$ induced an increase in acetylation of MT, a marker of MT stability, the speeds of $\mathrm{EB} 3$ comets in neurons treated with $\mathrm{A} \beta$, or with an HDAC6-specific inhibitor, tubacin, were faster than that in control neurons. In parallel, $A \beta$ increased the rate of catastrophe/pause, a phenomenon that might arise from the contemporary action of $\mathrm{A} \beta$ on actin retrograde flow that impose a force counteracting MT growth $^{41}$, indicating destabilization of MT as an overall effect, as MT undergo faster cycles of polymerization and depolymerization.

The apparent contradiction between hyperacetylated MT and destabilized MT was explained by biochemical analysis of tubulin population that indicated that, in the presence of $A \beta$, the increase 
a

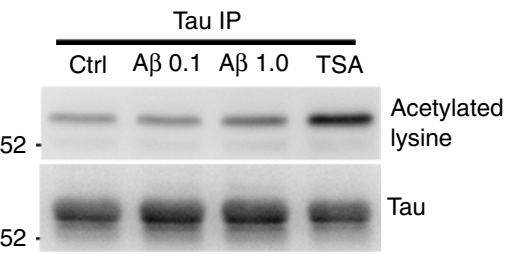

C

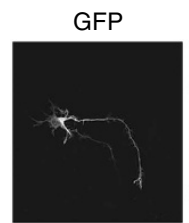

FLAG

$A \beta$
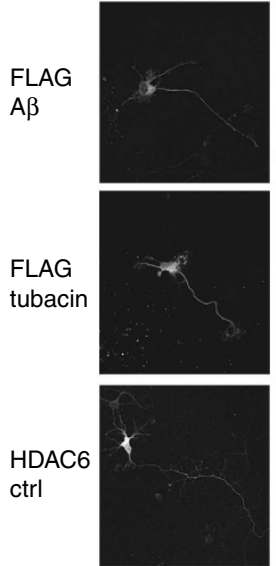

HDAC6
$A \beta$

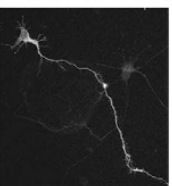

Tau
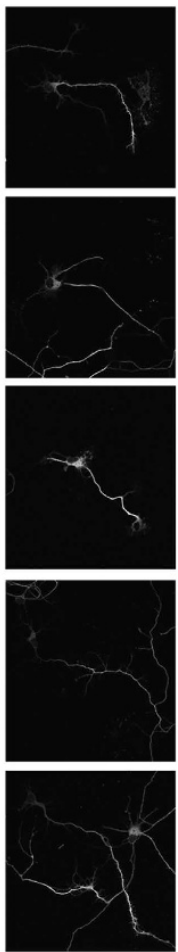

Merge
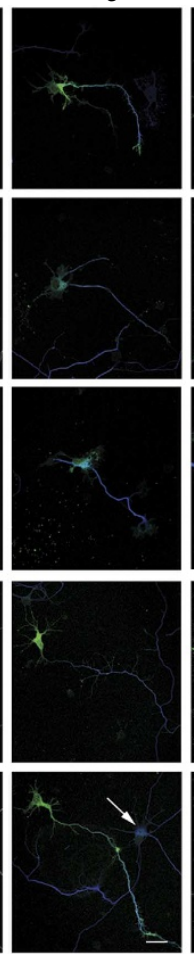

$4 \times$ merge
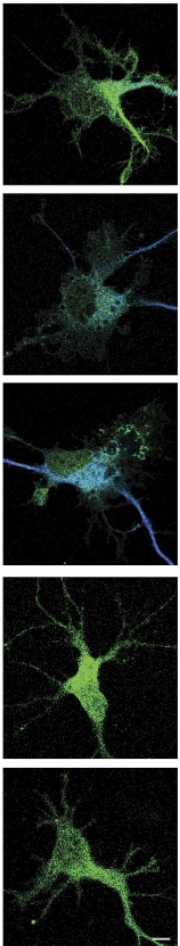

b

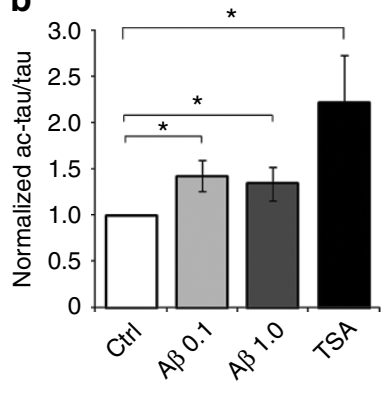

d

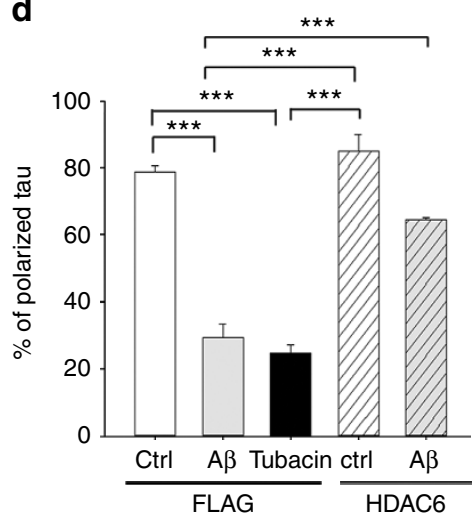

Figure 5 | A $\beta$ increases acetylated tau and induces missorting of tau. (a) Representative immunoblot showing acetylation of tau immunoprecipitated from lysates of cortical neurons treated with either $0.05 \%$ DMSO (ctrl), $100 \mathrm{nM} \mathrm{A} \beta$ (A $\beta$ 0.1), $1 \mu \mathrm{M} \mathrm{A} \beta$ (A $\beta$ 1.0) or 100 nM TSA. Immunoprecipitated tau (bottom panel) was overlaid for acetylated lysine (top panel). (b) Quantification of the ratio between the protein level of acetylated tau and total tau in tau immunoprecipitates from neurons treated with either $0.05 \%$ DMSO (ctrl, $1.00 \pm 0.00, n=7$ ), $100 \mathrm{nM} \mathrm{A \beta}(\mathrm{A} \beta 0.1,1.42 \pm 0.17, n=5), 1 \mu \mathrm{M} A \beta(\mathrm{A} \beta 1.0$, $1.34 \pm 0.18, n=4)$ or $100 \mathrm{nM}$ TSA (TSA, $2.22 \pm 0.52, n=7$ ). Statistical significance determined by two-tailed $t$-test. Ctrl/A $\beta 0.1:{ }^{*} P=0.012 ; c t r l / A \beta 1.0$ : ${ }^{\star} P=0.030$; ctrl/TSA: ${ }^{\star} P=0.038$. (c) Immunofluorescence analysis of tau localization (blue) in FLAG/GFP or HDAC6-FLAG/GFP-electroporated neurons (green), treated with either $0.05 \%$ DMSO (ctrl), $100 \mathrm{nM} \mathrm{A \beta}(A \beta)$ or $10 \mu \mathrm{M}$ tubacin (tubacin), showing missorting of tau in minor neurites in the presence of $A \beta$, which is prevented by HDAC6 overexpression. Note, in the bottom panels showing neurons treated with $A \beta$, the presence of a nonelectroporated neuron showing unpolarized tau staining (white arrow), and a neighbouring neuron overexpressing HDAC6 showing polarization of tau. Scale bar, $25 \mu \mathrm{m}$. Enlarged images of merged channels (far-right panels) showing minor neurites close to the cell body. Scale bar: $5 \mu \mathrm{m}$. (d) Quantification of the percentage of neurons showing polarized immunostaining of tau in axons, in neurons electroporated with FLAG/GFP and treated with either $0.05 \%$ DMSO (FLAG ctrl, 78.66 $\pm 1.95, n=91), 100 \mathrm{nM} \mathrm{A \beta}($ FLAG A $\beta, 29.26 \pm 4.16, n=94), 10 \mu \mathrm{M}$ tubacin (FLAG tubacin, 24.75 $\pm 2.53, n=20)$ or electroporated with HDAC6-FLAG/GFP and treated with 0.05\% DMSO (HDAC6 ctrl, $85.00 \pm 5.00, n=25$ ) or $100 \mathrm{nM} \mathrm{A \beta}$ (HDAC6 A $\beta, 64.32 \pm 0.68$, $n=31$ ). Statistical significance determined by one-way ANOVA, ${ }^{\star \star \star} P<0.001$. In $\mathbf{b}$ and $\mathbf{d}$, data are expressed as mean \pm s.e.m. and as mean percentage \pm s.e.m., respectively. ANOVA, analysis of variance.

in acetylation was attributable more to tubulin heterodimers than to MT. Moreover, the heterodimeric fraction was further increased due to MT instability and to the inhibitory effect of A $\beta$ on HDAC6. Increased myosin II-driven contractility together with MT instability in the axon would further exacerbate the tendency of the growth cone to collapse ${ }^{40}$, phenomenon that would affect growth cones motility in developing neurons or synapses stability in neuronal networks.

Our data on the accumulation of ac-tub heterodimers might explain controversial data in previous reports on the level of tubulin acetylation. For example, $\mathrm{AD}$ pathology, which displays destabilized MT, was classically linked with a reduction of $a c-t u b^{42}$, whereas a more recent study on tau interaction with
HDAC6 reported an increased level of ac-tub in AD samples ${ }^{43}$ Our results emphasize the importance of studying MT dynamics, besides tubulin post-translational modifications, in $\mathrm{AD}$ conditions when considering the use of HDAC6 inhibitors as a possible therapeutic approach for $\mathrm{AD}$. Although the use of inhibitors that target various members of HDAC family, such as valporic acid, sodium butyrate and TSA, were demonstrated to be neuroprotective and to improve memory processes, most of the studies attributed the neuroprotective mechanism to HDAC 1-4 and Sirtuins, and not specifically to HDAC6 (ref. 44). Controversial data on protective or neurotoxic effect of HDAC6 are present in the literature. It was shown that tubastatin $\mathrm{A}$, which specifically inhibits HDAC6, improved behavioural deficits, such 
a

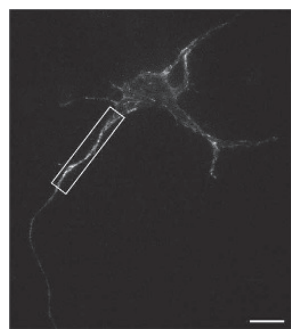

EB3-GFP

d
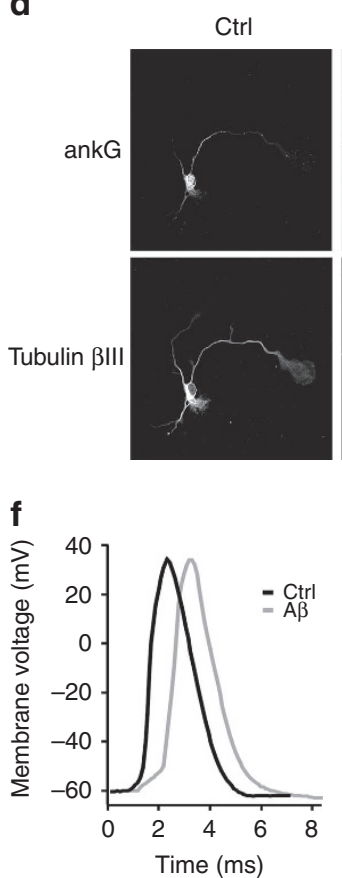

b

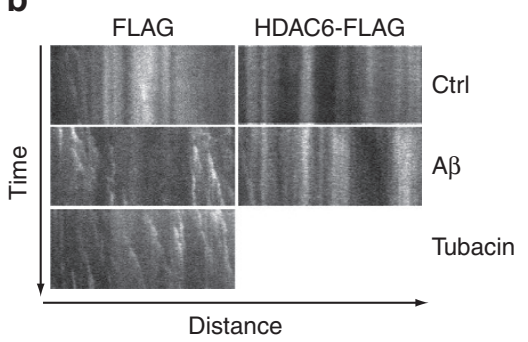

c

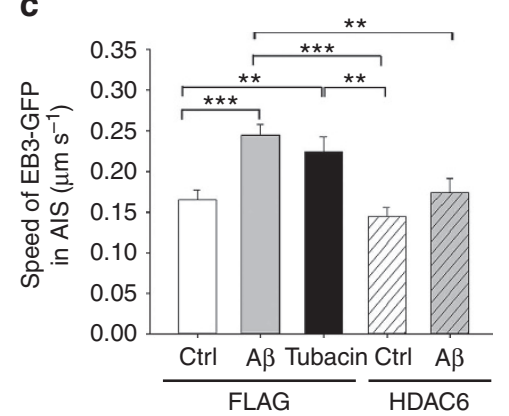

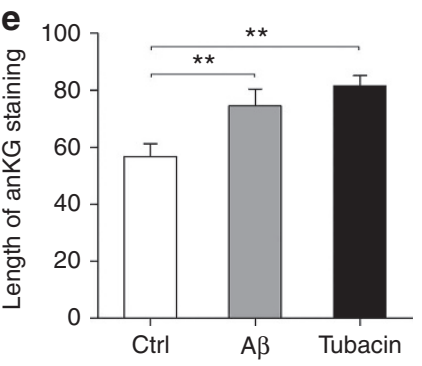

ox
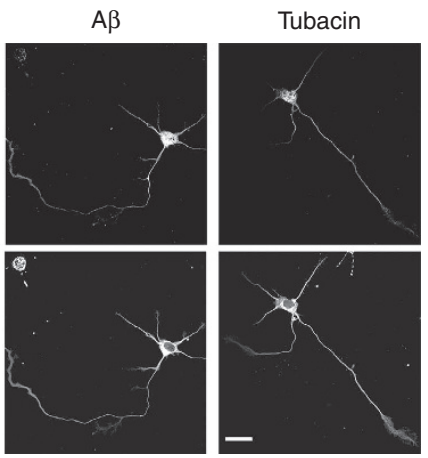

g

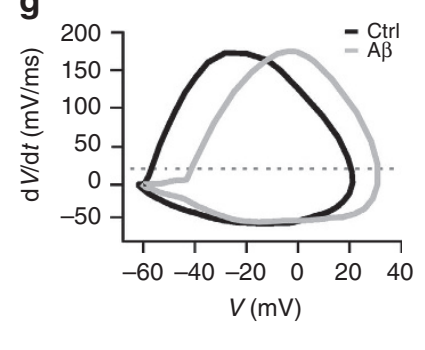

h

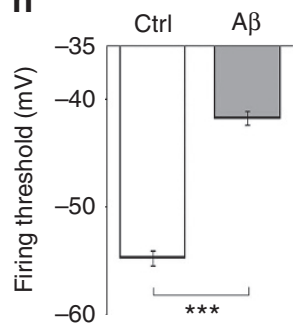

Figure 6 | A disrupts the retrograde barrier of tau by destabilizing MT in the AIS and the integrity of AIS by mislocalizing ankG. (a) Confocal image of a neuron electroporated with HDAC6-FLAG and EB3-GFP, showing the region corresponding to the AIS (white rectangle). Scale bar, $10 \mu \mathrm{m}$.

(b) Representative kymographs of the AIS of neurons co-electroporated with EB3-GFP and FLAG (left panels) or HDAC6-FLAG (right panels) and treated with $0.05 \%$ DMSO (ctrl), $100 \mathrm{nM} \mathrm{A \beta}(\mathrm{A} \beta)$ or $10 \mu \mathrm{M}$ tubacin (tubacin). The vertical arrow indicates the time ( $90 \mathrm{~s}$ ) and the horizontal arrow indicates the direction from the cell body to the growth cone. (c) Quantification of the velocity of EB3-GFP comets in the AIS in neurons co-electroporated with EB3-GFP/ FLAG (FLAG) and treated with either $0.05 \%$ DMSO (ctrl, $0.17 \pm 0.01, n=21), 100 \mathrm{nM} \mathrm{A} \beta$ for $1 \mathrm{~h}(\mathrm{~A} \beta, 0.24 \pm 0.01, n=11), 10 \mu \mathrm{M}$ tubacin for $1 \mathrm{~h}$ (tubacin, $0.22 \pm 0.02, n=10$ ) or electroporated with EB3-GFP/HDAC6-FLAG (HDAC6) and treated with $0.05 \%$ DMSO (ctrl, $0.14 \pm 0.01, n=11$ ) or $100 \mathrm{nM}$ A $\beta$ for $1 \mathrm{~h}(A \beta, 0.17 \pm 0.02, n=12)$. Statistical significance determined by one-way ANOVA, ${ }^{\star \star \star} P=0.001$ (FLAG ctrl/FLAG A $\beta$ ), ${ }^{\star \star} P=0.019(\mathrm{FLAG}$ ctrl/FLAG tubacin), ${ }^{\star \star \star} P<0.001$ (HDAC6 ctrl/FLAG A $\beta$ ), ${ }^{\star \star} P=0.005$ (HDAC6 ctrl/FLAG tubacin), ${ }^{\star \star} P=0.011$ (FLAG A $\beta /$ HDAC6 A $\beta$ ). (d) Immunofluorescence analysis of ankG and tubulin- $\beta$ III localization in neurons treated with either $0.05 \%$ DMSO (ctrl), $100 \mathrm{nM} \mathrm{A \beta}$ for $4 \mathrm{~h}$ (A $\beta$ ) or $10 \mu \mathrm{M}$ tubacin (tubacin). Scale bar, $25 \mu \mathrm{m}$. (e) Quantification of the length of ankG staining along the axon of neurons treated with either $0.05 \%$ DMSO (ctrl, $56.78 \pm 4.41, n=52$ ), $100 \mathrm{nM} \mathrm{A} \beta(\mathrm{A} \beta, 74.49 \pm 5.94, n=68)$ or $10 \mu \mathrm{M}$ tubacin $(81.57 \pm 3.67, n=42)$. Statistical significance determined by one-way ANOVA, ${ }^{\star \star} P=0.006$ (ctrl versus $A \beta$, ctrl versus tubacin). (f) Somatic current-clamp recording of a representative action potential evoked in neurons at 14 DIV treated for $4 \mathrm{~h}$ with $0.05 \%$ DMSO (black) or $100 \mathrm{nM} \mathrm{A \beta}$ (grey). (g) Phase plots of $\mathrm{dV} / \mathrm{d} t$ versus $V$ constructed from recording as in $\mathbf{f}$ in neurons treated for $4 \mathrm{~h}$ with $0.05 \%$ DMSO (black) or $100 \mathrm{nM} \mathrm{A \beta}$ (grey). (h) Quantification of the firing threshold of action potential in neurons treated with $0.05 \%$ DMSO (ctrl, $-51.33 \pm 1.83, n=10)$ or $A \beta(A \beta,-33.82 \pm 2.81, n=10)$. Statistical significance determined by two-tailed $t$-test, ${ }^{\star \star \star} P<0.001$. In $\mathbf{c}, \mathbf{e}$ and $\mathbf{h}$, data are expressed as mean \pm s.e.m. ANOVA, analysis of variance.

as hyperactivity, fear conditioning and spatial navigation memory deficits in a transgenic mouse model of tauopathy ${ }^{45}$. A recent study correlated the amelioration of memory function induced by HDAC6 deletion in a model of $\mathrm{AD}$, with the rescue in mitochondrial trafficking ${ }^{46}$. HDAC6 inhibition also improved the impaired mitochondrial transport in a model of CharcotMarie-Tooth disease ${ }^{47}$, and nonspecific HDAC inhibitors, such as suberoylanilide hydroxamic acid and TSA, application alleviates the impaired axonal transport of vesicles by enhancing the interaction between kinesin-1 and MT in a mouse model of Huntington's disease ${ }^{48}$.

On the other hand, another group showed that genetic depletion of HDAC6 did not modify the progression of Hungtinton's disease ${ }^{49}$. It was also shown that phosphorylation of HDAC6, activating its deacetylase activity, promotes proper aggresome formation and protects from toxicity ${ }^{50}$. Indeed, HDAC6 inhibition would impair the removal of aggregated protein by retrograde transport to autophagosomes 
and lysosomes as it was shown that tubacin treatment decreased the recruitment of Atg/LC3, a crucial component of autophagic transport ${ }^{51}$, and that HDAC6 expression rescued neurodegeneration in a model of spinal and bulbar muscular atrophy ${ }^{52}$. HDAC6 has been shown also to protect dopaminergic neurons from alpha-synuclein-induced toxicity ${ }^{53}$.

For what concerns axonal pathology, MT stability must be regulated at an optimal level to allow axon and dendrites development in neuritogenesis, and it has been demonstrated that HDAC6 is required to provide efficient MT deacetylation ${ }^{54}$. Consistently, it has been shown that silencing of HDAC6 reduced neurite outgrowth and that its retransfection was able to revert to the normal phenotype ${ }^{55}$. It is thus critical to consider the deacetylase isoforms, and the specific function defective in the diseases, to evaluate HDACs or HDACs inhibitors for therapeutic intervention. Acetylation occurs on lysine, the same amino acid on which ubiquitination for protein degradation takes place. Further experiments would be needed to study if acetylated heterodimeric tubulin can still be ubiquitinated by ubiquitin ligases ${ }^{56}$.

Tau interacts and is deacetylated by HDAC6 (ref. 57), in fact TSA treatment leads to dramatic accumulation of ac-tau, and ac-tau was found in NFTs in various neurodegenerative diseases $^{16,17}$. Phosphorylation of tau, a modification of tau that underlies tau-mediated degeneration, and that was shown to impair synaptic function at preclinical stages of disease ${ }^{58}$, has been associated with this acetylation state. Indeed, tau acetylation was shown to prevent the degradation of phosphorylated tau ${ }^{17}$. Both forms have impaired MT binding, which results in further MT instability ${ }^{16}$. A recent study in oligodendrocytes showed that inhibition of HDAC6 caused an increase in hyperphosphorylated tau that disrupts its interaction with $\mathrm{MT}^{59}$.

Interestingly, a recent report showed that mutant tau associated with tauopathies is sequestered by its binding to heterodimeric tubulin, suggesting a role of unpolymerized tubulin in $\mathrm{AD}$ and other tau pathologies ${ }^{60}$. Moreover, overexpression of tau was shown to increase ac-tub in fibroblasts ${ }^{61}$, which may be explained by tau-driven inhibition of HDAC6 (ref. 43). This gives rise to the possibility that polarized cells, such as neurons, which express high level of tau and ac-tub, may be more susceptible to changes in tau and/or tubulin levels induced by $A \beta$ in comparison with other cell types.

It is noteworthy that both substrates of HDAC6, ac-tub and tau, are enriched in axons, suggesting a role of HDAC6 in neuronal polarity. Here we provide evidence that maintenance of polarized axonal identity is disrupted by $A \beta$ in an HDAC6dependent manner. Importantly, overexpression of HDAC6 was sufficient to prevent the effect of $\mathrm{A} \beta$, and, together, with a decrease in ac-tub and tau, a function of HDAC6 as a MAP 20,22 may be implicated in the maintenance of stabilized MT in the AIS. Neuron polarization consists of morphological polarization that involve preferential elongation of the axon; sorting of proteins in different compartments; and functional polarization, in which the AIS barrier is also involved. It is a very complex phenomenon that requires local destabilization of $\operatorname{actin}^{62}$ and local stabilization of MTs ${ }^{63}$. RhoA activation is known to decrease actin turnover, stabilizing actin, and thus participating in axon retraction ${ }^{64}$. Moreover, human mutant tau has the ability to bind and bundle F-actin in vitro ${ }^{65}$. A $\beta$-driven actin stabilization and MT destabilization are therefore both possibly hampering neuronal elongation and polarization, not excluding the possibility of a mechanistic link mediated by other players. Finally, we showed that ankG and sodium channels are dispersed along the axon on exposure to $A \beta$. Consistently, a recent report showed defective filtering at the axon initial segment in cultured neurons from an $\mathrm{AD}$ mouse model ${ }^{66}$. In conclusion, as summarized in the model shown in Fig. 7, our study demonstrates that extracellular $A \beta$ and intracellular $A \beta$ target RhoA and HDAC6, respectively, altering actin and MT dynamics. The activity of $\mathrm{A} \beta$ on its targets leads to increased actin retrograde flow, instability of MT and hyperacetylation and missorting of tau. The combined effects on cytoskeletal dynamics and acetylation levels induce loss of AIS integrity and impairment in action potential firing, implicating RhoA and HDAC6 in the interplay between $\mathrm{A} \beta$ and tau in $\mathrm{AD}$.

\section{Methods}

Peptides. $\beta$-Amyloid (1-42), HiLyte Fluor 488-labelled $\beta$-amyloid (1-42) and scrambled $\beta$-amyloid (1-42) were purchased from Anaspec, Inc. (Fremont, CA USA). The peptides were solubilized in $100 \%$ dimethylsulphoxide, aliquoted and kept at $-80^{\circ} \mathrm{C}$.

Reagents. Blebbistatin, TSA, tubacin and taxol were purchased from SigmaAldrich (St Louis, MO, USA). Y27632 was purchased from Millipore Corporation (Billerica, MA, USA).

Expression plasmids. pRaichu- $1298 \times$ was a kind gift from Professor Michiyuki Matsuda (Kyoto University, Kyoto, Japan); pEGFP-N1-LifeAct was a kind gift from Dr Giorgio Scita (IFOM, Milan, Italy); pEGFP-N1 was purchased from Clonetech Laboratories, Inc. (Mountain View, CA, USA); pCMV-AC-GFP-EB3-GFP was purchased from Origene Technologies, Inc. (Rockville, MD, USA); pCDNA3.1HDAC6-FLAG-was purchased from Addgene, Inc. (Cambridge, MA, USA); and pCMV-FLAG-3 $\times$ was purchased from Sigma-Aldrich.

Antibodies. The antibodies used were as following: monoclonal anti-ac-tub and anti-pan- $\mathrm{Na}^{+}$channel (Sigma-Aldrich); anti-tubulin- $\beta$ III, anti-MAP2 and anti-A $\beta$ 6E10 (Covance, Princeton, NJ, USA); anti-acetylated lysine (Cell Signaling Technology Inc., Danvers, MA, USA); anti-ankG (Life Technologies, Paisley, UK); anti-tau: Tau-1 and Tau-5 (Merck Millipore, Billerica, MA, USA); and polyclonal anti-tubulin- $\beta$ III (Sigma-Aldrich). All antibodies were used at a dilution of 1:1,000 for immunoblotting experiments and of 1:100 for immunoistochemistry experiments.

Neuronal culture. All experiments were performed in accordance with Italian Ministry of Health. Primary cultures were obtained from hippocampi or cortices derived from embryonic day 18 C57BL6J mice (Harlan Laboratories Inc., Indianapolis, IN, USA). Embryos were removed and dissected under sterile conditions. Cortices and hippocampi were dissociated by enzymatic digestion in $0.125 \%$ trypsin-EDTA (Life Technologies) for $30 \mathrm{~min}$ at $37^{\circ} \mathrm{C}$ and cortices were further treated with $0.25 \mathrm{mg} \mathrm{ml}^{-1}$ DNase in Hank's balanced saline solution (Life Technologies) $/ 2 \mathrm{mM}$ calcium chloride (Sigma-Aldrich) for $30 \mathrm{~min}$ at $37^{\circ} \mathrm{C}$. Trypsin activity was blocked by adding complete medium, which consists of Neurobasal Media (Life Technologies) supplemented with 2\% B27 (Life Technologies), 2 mM GlutaMax (Life Technologies), $100 \mathrm{U} \mathrm{ml}^{-1}$ penicillin-streptomycin (Life Technologies) and $10 \%$ fetal bovine serum (FBS; Life Technologies). After trypsinization, hippocampi were rinsed in complete medium without FBS, dissociated with a plastic pipette, and 20,000-40,000 hippocampal neurons were plated at a concentration of $0.20-1 \times 10^{5}$ cells per millilitre on

18 -mm diameter coverslips precoated with $0.1 \mathrm{mg} \mathrm{ml}^{-1}$ poly-D-lysine (SigmaAldrich). After trypsinization, cortices were rinsed in the complete media without FBS, dissociated with a plastic pipette and centrifuged at $1,500 \mathrm{~g}$ for $7 \mathrm{~min}$, and $2 \times 10^{6}$ cortical neurons were plated $60-\mathrm{mm}$ diameter Petri dishes precoated with $0.01 \mathrm{mg} \mathrm{ml}^{-1}$ poly-D-lysine (Sigma-Aldrich).

Atomic force microscopy. AFM imaging was performed employing a Nanowizard II (JPK instruments, Germany) in intermittent contact mode, in air. Single-beam uncoated silicon cantilevers (OMCL-AC160TS-W, Olympus, Japan) with typical resonance frequency of $300 \mathrm{kHz}$ were used. The nominal tip radius of curvature of the tip is $10 \mathrm{~nm}$. Fifteen microlitres aliquots of $12 \mu \mathrm{M}$ A $\beta$ incubated for different times in Neurobasal were deposited on freshly cleaved mica substrates and allowed to adhere for $10 \mathrm{~min}$, before washing with water and drying under a gentle $\mathrm{N}_{2}$ stream.

Immunocytochemistry and confocal microscopy. Primary hippocampal neurons cultured on coverslips were fixed in $4 \%$ paraformaldehyde/30\% glycerol in PBS for $7 \mathrm{~min}$. The neurons immunostained for ankG were treated with cold $100 \%$ methanol for $5 \mathrm{~min}$ at $-20^{\circ} \mathrm{C}$ after fixation. The coverslips were washed in PBS four times and were incubated with primary antibodies. After four washes in $20 \mathrm{mM}$ phosphate buffer with $0.5 \mathrm{M} \mathrm{NaCl}$, fluorescently conjugated secondary antibodies in $5 \%$ normal goat serum $/ 0.1 \%$ Triton in PBS were added to the coverslips. The coverslips were washed in $20 \mathrm{mM}$ phosphate buffer with $0.5 \mathrm{M}$ 

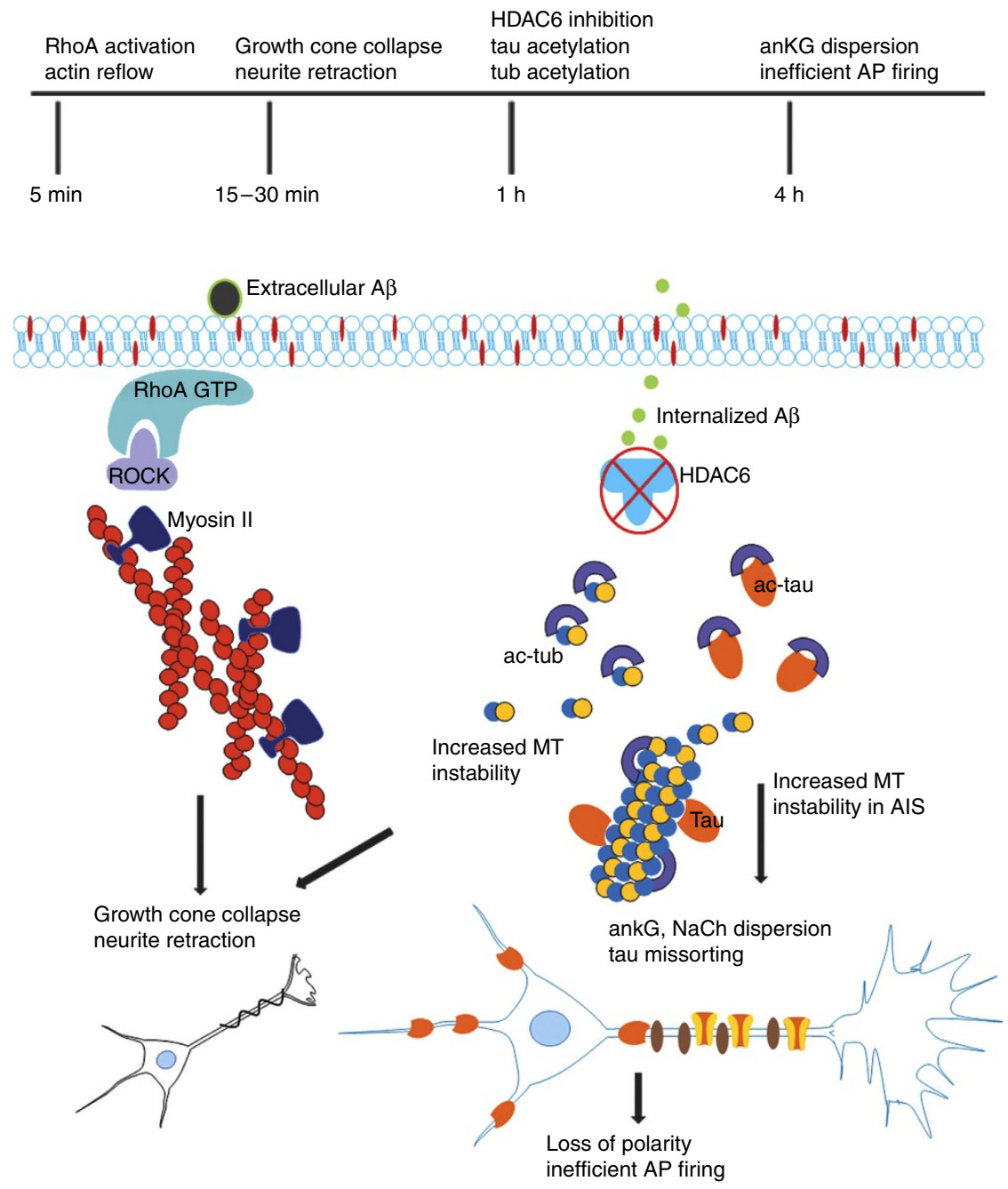

Figure 7 | Model of A $\mathbf{A}$ 's mechanisms of action. The upper part illustrates a hypothetical time course showing short- and long-term effects of $A \beta$ in our experimental conditions. The lower part shows a cartoon of the combined effects of extracellular and intracellular $A \beta$. Extracellular $A \beta$, which is kept in contact with the cell surface, activates RhoA and its downstream targets ROCK and myosin II, increasing actin retrograde flow. Internalized soluble A $\beta$ inhibits HDAC6 inducing an increase in the acetylation of tubulin heterodimers. In parallel, tau is also hyperacetylated due to HDAC6 inhibition, and MT instability is favoured. The alterations of the dynamics of both cytoskeletal elements induce growth cone collapse and neurite retraction. MT instability in the AIS in particular leads to dispersion of ankG (brown ovals) and of $\mathrm{Na}^{+}$channels (yellow channels) outside the AIS, and to missorting of tau in dendrites, with ensuing loss of neuronal polarity and inefficient AP firing.

$\mathrm{NaCl}$ four times, once in PBS and dipped in distilled water before being mounted in ProLong Gold Antifade Reagent (Life Technologies). Confocal images were acquired on a Leica TCS SP5 microscope using a $63 \times$ objective (Leica Microsystems, Wetzlar, Germany).

Axonal length. Primary hippocampal neurons plated on $18-\mathrm{mm}$ diameter coverslips were incubated with $1 \mu \mathrm{M}$ A $\beta$ for $1 \mathrm{~h}$. The neurons were immunostained with anti-tubulin- $\beta I I I$ as described above. All neurites were traced using NeuronJ plug-in in ImageJ, and the length of the longest neurite, considered as axon, was measured using ImageJ.

Electroporation of primary hippocampal neurons. Neurons $\left(1 \times 10^{6}\right)$ were electroporated with $1 \mu \mathrm{g}$ of DNA using P3 Primary Cell 4D-Nucleofector Kit and the pulse code CU133 on the 4D-Nucleofector System (Lonza Group Ltd, Basel, Switzerland).

Coating of microspheres with beta-amyloid peptides. Three micrograms of carboxyl polymer microspheres of $4.08 \mu \mathrm{m}$ diameter (Bangs Laboratories Inc., Fishers, IN, USA) were washed and resuspended in coupling buffer from Poly-Link Coupling Kit for (Polysciences Inc., Warrington, PA, USA) containing $0.022 \mathrm{mg} \mu \mathrm{l}^{-1}$ of 1,3 -propanediamine, $N^{\prime}$-(ethylcarbonimidoyl)- $N, N$-dimethylmonohydrochloride (EDAC) for $2 \mathrm{~h}$ at room temperature. EDAC solution was removed by centrifugation at $1,000 \mathrm{~g}$ for $10 \mathrm{~min}$, the microspheres were washed in the coupling buffer without EDAC. The activated microspheres were resuspended in
$10 \mathrm{mM}$ phosphate buffer at $\mathrm{pH} 7.4$ containing $1 \mu \mathrm{g} \mu \mathrm{l}^{-1} \beta$-amyloid (1-42), HiLyte Fluor 488-labelled $\beta$-amyloid (1-42) or scrambled- $\beta$-amyloid (1-42) (Anaspec Inc.) incubated at $4{ }^{\circ} \mathrm{C}$ overnight. Excess unbound peptide was removed by centrifugation at $1,000 \mathrm{~g}$ for $5 \mathrm{~min}$ at $4^{\circ} \mathrm{C}$ and the microspheres were resuspended in $10 \mathrm{mM}$ phosphate buffer to the concentration of $0.06 \mu \mathrm{g} \mathrm{Il}^{-1}$. Microspheres coated with the desired peptide were resuspended in the conditioned medium from the neuronal culture at a concentration of $0.04 \mu \mathrm{g} \mu \mathrm{l}^{-1}$, and a total of $0.4 \mu \mathrm{g}$ was added to the neuron dish placed in the optical setup equipped with an incubator maintained at $35^{\circ} \mathrm{C}$ with $5 \% \mathrm{CO}_{2}$ balanced with air ${ }^{67}$.

Force spectroscopy measurements. We applied interferometric tracking of a functionalized microsphere held in an optical trap and brought in contact with the neck of the axonal growth cone of a mouse neuron. The optical system to perform long-term force spectroscopy measurements was previously described in detail ${ }^{25}$. Briefly, it consists of a modified upright microscope integrating an optical tweezers system working in force-clamp condition (with force equal to zero). The position of the bead with respect to the centre of the trap ( $x_{\mathrm{QPD}}, y_{\mathrm{QPD}}$ and $\left.z_{\mathrm{QPD}}\right)$ is measured through a QPD. During the interaction with the cell, the system is operated in forceclamp with a $0 \mathrm{pN}$ set point. To keep the bead in the centre of the trap, the QPD signal is fed back to a proportional-integral stage driving the coarse motion of a three axis piezo stage (PIEZO) in space ( $S x, S y$ and Sz). QPD and PIEZO signals were sampled independently and simultaneously at a $2 \mathrm{kHz}$ acquisition rate, while brightfield images were acquired at $1 \mathrm{~Hz}$ frame rate and synchronized in time with the data. 
For every experiment, the bead was positioned in contact with the neck of the growth cone, the region connecting the growth cone with the axon shaft. Supplementary Figure 2a shows a typical example of a growth cone rearranging its shape around the trapped microsphere, while the corresponding spatial trajectory (Sx, Sy and Sz) and QPD signals (forces) are reported in Supplementary Fig. 2b. A complementary representation of the same experiment is reported in Supplementary Fig. $2 \mathrm{c}$ in which the shift of the microsphere on the membrane apical surface is shown by the cyan line, and the random forces acting on the microsphere are represented as vector field (coloured arrows). To obtain this representation, average force acting on the microsphere was calculated by vectoraveraging the displacement from the trap centre over a time window of $1 \mathrm{~s}$, multiplied by the calibrated trapping stiffness (that was kept constant along the whole experiment).

Independent information can be obtained by analysis of the QPD and PIEZO signals. The QPD traces encode higher frequencies that are associated with the Brownian motion of the cell-adhered probe (in $x, y$ and $z$ directions). When the bond between the cell and the bead tightens, the average motion of the bead is reduced, and is indicated by a measurable drop in the variance of the signal, $\operatorname{var}_{\mathrm{QPD}}$ (Supplementary Fig. 2d). The variance of the QPD motion was computed as the total variance of $x_{\mathrm{QPD}}, y_{\mathrm{QPD}}$ and $z_{\mathrm{QPD}}$ traces on a time-shifted window of 6,000 points, with half-time window (3,000 points) overlap (the variance is computed each $1.5 \mathrm{~s}$ ). The decrease of the variance of cell-adhered bead $\Delta \mathrm{var}=\operatorname{var}_{\mathrm{QPD}, t=0}-\operatorname{var}_{\mathrm{QPD}, t=\text { end }}(t=$ end, indicate the last acquired time point $)$ was calculated with respect to the value of the first second of recording and normalized to the total duration of the experiment: $\Delta \mathrm{var} / \Delta t$ (typical duration of an experimental session is $45 \mathrm{~min}$ ).

Alongside, PIEZO signals encode lower frequency information associated with the overall motion of the probe on the apical surface of the growth cone, and thus on the local remodelling of the leading edge of the cell, through cytoskeleton and molecular motor dynamics ${ }^{68}$. The vertical position of the bead, $\mathrm{Sz}$ (see an example in Supplementary Fig. 2e), was taken as a direct measure of the growth cone state, and its shrinking on local application of coated microsphere was quantified in terms of the total vertical displacement: $\Delta \mathrm{S} z=z_{\mathrm{PIEZO}, t=0}-z_{\mathrm{PIEZO}, t=\text { end }}$. Moreover, the in-plane velocity of the bead was calculated by interpolation of the spatial trajectory ( $S x$ and $S y$ ) over a time interval of $100 \mathrm{~ms}$ (that is, 200 points window) and the modulus $V=\left(V_{\mathrm{S} x}\right)^{2}+\left(V_{\mathrm{S} y}\right)^{2}$ was taken as a reference of the speed of the bead on the cell surface.

FRET analysis. Microsphere coated as described above was attached to a neurite of 3 DIV hippocampal neurons electroporated with pRaichu- $1298 \times$ and time-lapse images were taken every $20 \mathrm{~s}$ for a total of $30 \mathrm{~min}$. FRET probes were excited with a TTL modulated 405-nm laser source (1-s light pulse duration), and the emitted fluorescence light was collected and separated through a dichroic mirror and sent to two synchronized charge-coupled devices (CCDs; acquiring the distinct donoracceptors signals). The CCDs integrated the emitted photons during the $20 \mathrm{~s}$ time frame period. Therefore, we could reduce the light exposure, and avoid the unwanted photobleaching of donor-acceptor dyes. To quantify the FRET efficiency, we register the two acquired stack trough image cross-correlation (Huygens software), we subtracted the background signals and detect the cell area trough image segmentation (ImageJ). Then, we compute the ratio imaging on the identified cell region of interest in the field of view. FRET ratio where normalized to the baseline value prestimulation. To compute the FRET efficiency increase, we calculate the averaged FRET value on the stimulated axon region of interest for each time frame.

Live imaging of actin retrograde flow. Hippocampal neurons from embryonic day 18 mouse embryos prepared as described above were plated onto a glass bottom dish (MatTek Corporation, Ashland, MA, USA) at 2,000 neurons per millimetre. A coated microsphere was optically driven in contact with the neck of a growth cone, and a time-lapse movie of LifeAct-GFP was acquired. Fluorochromes were excited with a TTL modulated (200-ms light pulse duration) 473-nm laser source (TECBL-15 G-473-TTL-FC, World Star Tech. Inc., USA), and the emitted fluorescence was integrated during the 1-s time frame period through the CCD (V887 ECS UVB EMCCD, Andor, Italy). Kymographs of LifeAct-GFP time-lapse movies were constructed using MultipleKymograph plug-in of ImageJ.

Live imaging and EB3-GFP tracking. Time-lapse images of GFP comets in mouse hippocampal neurons electroporated with EB3-GFP were taken in total internal reflection mode set at $250 \mathrm{~nm}$ on Leica TCS SP5 microscope with a $100 \times$ objective (Leica Microsystems, Wetzlar, Germany) at 1 frame per second for $90 \mathrm{~s}$. For MT dynamics neurons at 3 DIV were used, whereas neurons at 5 DIV were used for MT dynamics in the AIS. The speed of the GFP comets was analysed using the object analyser in Huygens Professionals v.3.7 (Scientific Volume Imaging B.V., Hilversum, The Netherlands). Kymographs of EB3-GFP time-lapse movies were constructed using MultipleKymograph plug-in of ImageJ. Number of catastrophes/ pause per MT were counted as gaps in the EB3-GFP lines in the kymographs, which were created from the corresponding time-lapse movies using Image J as described above

In vitro assay of HDAC6 catalytic activity. Catalytic activity of HDAC6 was assayed in the presence of $0.05 \%$ DMSO, $50 \mathrm{nM}$ TSA, $100 \mathrm{nM} \mathrm{A} \beta$ or $1 \mu \mathrm{M} \mathrm{A} \beta$ using
Fluor-de-Lys HDAC6 fluorometric drug discovery kit (Enzo Life Sciences, Inc., Farmingdale, NY, USA) according to the manufacturer's instructions.

Separation of tubulin and MTs. Cortical neurons $\left(2 \times 10^{6}\right)$ were fixed with $4 \%$ paraformaldehyde in PBS for $1 \mathrm{~min}$. After fixation neurons were homogenized in $200 \mu \mathrm{l}$ of PBS, with $0.1 \%$ Triton X-100, protease inhibitor cocktail (Roche, Basel, Switzerland) and phosphatase inhibitor cocktails 2 and 3 (Sigma-Aldrich) by passing through needle of $28 \mathrm{G} \times 1 / 2$ in (BD, Franklin Lakes, NJ, USA) for five passages at room temperature. The homogenates were centrifuged at $1,000 \mathrm{~g}$ to remove cell debris and the proteins were separated by $7 \%$ SDS-polyacrylamide gel electrophoresis (SDS-PAGE).

Immunoblotting. Following 10\% SDS-PAGE, proteins were transferred onto nitrocellulose membrane, blocked in 5\% milk/TBS/Tween $0.05 \%$ for $1 \mathrm{~h}$ at room temperature and incubated with primary antibodies for $2 \mathrm{~h}$ at room temperature. The membranes were washed four times for a total of $30 \mathrm{~min}$ in TBS/ Tween $0.05 \%$ and incubated with horseradish peroxidase-conjugated secondary antibody for $1 \mathrm{~h}$ at room temperature. The membranes were washed four times for a total of $30 \mathrm{~min}$ in TBS/Tween $0.05 \%$, overlayed with Amersham ECL Western Blotting Detection Reagent (GE Healthcare Life Sciences, Freiburg, Germany) and imaged for chemiluminescence using ImageQuant LAS 500 (GE Healthcare Life Sciences).

Permeabilization before immunocytochemistry. Primary hippocampal neurons cultured on coverslips were gently covered with MSB with $0.1 \%$ Triton $\mathrm{X}-100$ for $10 \mathrm{~s}$ at room temperature before fixation in $4 \%$ paraformaldehyde $/ 4 \%$ sucrose in PBS. Following the fixation, the neurons were immunostained as described above.

Tau immunoprecipitation. Cortical neurons $\left(2 \times 10^{6}\right)$ were homogenized in $100 \mu \mathrm{l}$ of $\mathrm{MSB} / 150 \mathrm{mM} \mathrm{NaCl} / 0.5 \%$ Triton-X with protease inhibitor cocktail (Roche) and phosphatase inhibitor cocktails 2 and 3 (Sigma-Aldrich) by passing through needle of $28 \mathrm{G} \times 1 / 2$ in (BD) for 10 passages at room temperature and left on ice for $30 \mathrm{~min}$. Twenty microgram of total protein was incubated with $1 \mu \mathrm{g}$ of tau-1 antibody for $2 \mathrm{~h}$ at $4{ }^{\circ} \mathrm{C}$. Antibody-bound fractions were pulled down with Protein G-Sepharose 4B (GE Healthcare Life Sciences), washed with MSB containing $0.1 \%$ Triton-X twice before eluting in the laemmli buffer and boiled at $100^{\circ} \mathrm{C}$ for $5 \mathrm{~min}$. The immunoprecipitates were separated by SDS-PAGE and immnoblotted using as described above.

Electrophysiological recordings. Action potentials were recorded in the whole-cell configuration of the patch-clamp technique. The extracellular recording solution ( $145 \mathrm{mM} \mathrm{NaCl} / 2 \mathrm{mM} \mathrm{KCl} / 2 \mathrm{mM} \mathrm{MgCl} / 2 / 1 \mathrm{mM} \mathrm{CaCl}_{2} / 10 \mathrm{mM}$ glucose $/ 10 \mathrm{mM}$ HEPES, pH 7.4), patch pipettes pulled from borosilicate glass capillaries (Hilgenberg, Malsfeld, Germany) had a $4-5 \mathrm{M} \Omega$ resistance when filled with intracellular recording solution ( $130 \mathrm{mM} \mathrm{K}$-gluconate $/ 5 \mathrm{mM} \mathrm{KCl} / 5 \mathrm{mM}$ sucrose $/ 1 \mathrm{mM}$ EGTA/10 mM HEPES/4 mM Mg-ATP, $300 \mathrm{mOsm}$ and pH 7.2 with $\mathrm{KOH}$ ). Cells were current clamped at about $-65 \mathrm{mV}$ and the action potentials evoked were acquired using Clampex 10.0 software (Molecular Devices, Sunnyvale, CA, USA). During the experiments, the stability of the patch was checked by continuously monitoring the series resistance: cells exhibiting $10-15 \%$ changes were excluded from the analysis. Currents were sampled at $10 \mathrm{kHz}$ and digitally filtered at $3 \mathrm{kHz}$ using the $700 \mathrm{~B}$ Axopatch amplifier (Molecular Devices). During the experiments, neurons were continuously perfused at $12 \mathrm{ml} \mathrm{h}^{-1}$ with the extracellular recording solution.

Statistical analysis. Two-tailed tests were performed using SigmaPlot 12.0 (Systat Software, Inc., San Jose, CA, USA) for comparison of two groups and expressed as mean values \pm s.e.m. For all pair-wise multiple comparison procedure, Holm-Sidak method was used for one-way analysis of variance using SigmaPlot 12.0 (Systat Software), and the data were expressed as mean values \pm s.e.m. $P$-values $<0.05$ were considered to be statistically significant. The power of performed test with alpha $(\pi)$ for the statistical analysis and when the $t$-tests were used, the $t$-values, the degrees of freedom (df) are also included as follows: Fig. 1a: $t=6.170, \mathrm{df}=291$, $\pi=0.050: 1.000$; Fig. 1e: $c$ ctrl versus $\mathrm{A} \beta: t=5.450, \mathrm{df}=10, \pi=0.050: 0.999 ; \mathrm{A} \beta$ versus $\mathrm{A} \beta+\mathrm{Y} 27632: t=-2.547, \mathrm{df}=8, \pi=0.050: 0.536 ; \mathrm{A} \beta$ versus $\mathrm{A} \beta+$ blebb: $t=-3.546, \mathrm{df}=7, \pi=0.050: 0.849$; Fig. 2a: $\pi=0.050: 0.711$; Fig. 2b: $\pi=0.050: 0.966$; Fig. 2f: $t=-3.837, \mathrm{df}=14, \pi=0.050: 0.944$; Fig. 2i: $t=-3.359$, $\mathrm{df}=86, \pi=0.050: 0.906$; Fig. 3b: $\pi=0.050: 0.854$; Fig. $3 \mathrm{c}: \pi=0.050: 0.961$; Fig. $4 \mathrm{~b}$ : $\pi=0.050: 0.486$; Fig. 4c: $\pi=0.050: 1.000$; Fig. 4e: MT: $\pi=0.050: 1.000$, monomers: $\pi=0.050: 0.962$; Fig. 4f: $t=-3.549, \mathrm{df}=59, \pi=0.050: 0.935$; Fig. $5 \mathrm{~b}$ : ctrl versus $\mathrm{A} \beta 0.1: t=-3.053, \mathrm{df}=10, \pi=0.050: 0.747$; ctrl versus $\mathrm{A} \beta 1.0: t=2.573, \mathrm{df}=9$, $\pi=0.050: 0.559$; $\mathrm{ctrl}$ versus TSA: $t=-1.218, \mathrm{df}=12, \pi=0.050: 0.482$; Fig. $5 \mathrm{~d}$ : $\pi=0.050: 1.000$; Fig. 6c: $\pi=0.050: 0.994$; Fig. 6e: $\pi=0.050: 0.744$; Fig. 6h: ctrl versus $\mathrm{A} \beta: t=-5.210, \mathrm{df}=18, \pi=0.050: 0.999$. 


\section{References}

1. Masliah, E. The role of synaptic proteins in Alzheimer's disease. Ann. N. Y. Acad. Sci. 924, 68-75 (2000).

2. Mucke, L. et al. High-level neuronal expression of abeta 1-42 in wild-type human amyloid protein precursor transgenic mice: synaptotoxicity without plaque formation. J. Neurosci. 20, 4050-4058 (2000).

3. Meberg, P. J. \& Bamburg, J. R. Increase in neurite outgrowth mediated by overexpression of actin depolymerizing factor. J. Neurosci. 20, 2459-2469 (2000).

4. Matus, A. Actin-based plasticity in dendritic spines. Science 290, 754-758 (2000)

5. Minamide, L. S., Striegl, A. M., Boyle, J. A., Meberg, P. J. \& Bamburg, J. R Neurodegenerative stimuli induce persistent ADF/cofilin-actin rods that disrupt distal neurite function. Nat. Cell Biol. 2, 628-636 (2000).

6. Maloney, M. T., Minamide, L. S., Kinley, A. W., Boyle, J. A. \& Bamburg, J. R. Beta-secretase-cleaved amyloid precursor protein accumulates at actin inclusions induced in neurons by stress or amyloid beta: a feedforward mechanism for Alzheimer's disease. J. Neurosci. 25, 11313-11321 (2005)

7. Price, D. L., Tanzi, R. E., Borchelt, D. R. \& Sisodia, S. S. Alzheimer's disease: genetic studies and transgenic models. Annu. Rev. Genet. 32, 461-493 (1998)

8. Rapoport, M., Dawson, H. N., Binder, L. I., Vitek, M. P. \& Ferreira, A. Tau is essential to beta -amyloid-induced neurotoxicity. Proc. Natl Acad. Sci. USA 99, 6364-6369 (2002).

9. Roberson, E. D. et al. Reducing endogenous tau ameliorates amyloid betainduced deficits in an Alzheimer's disease mouse model. Science 316, 750-754 (2007)

10. Vossel, K. A. et al. Tau reduction prevents Abeta-induced defects in axonal transport. Science 330, 198 (2010).

11. Zempel, H., Thies, E., Mandelkow, E. \& Mandelkow, E. M. Abeta oligomers cause localized $\mathrm{Ca}(2+)$ elevation, missorting of endogenous Tau into dendrites, Tau phosphorylation, and destruction of microtubules and spines. J. Neurosci. 30, 11938-11950 (2010).

12. Zempel, H. \& Mandelkow, E. M. Linking amyloid-beta and tau: amyloid-beta induced synaptic dysfunction via local wreckage of the neuronal cytoskeleton. Neurodegener. Dis. 10, 64-72 (2012).

13. Walsh, D. M. et al. Naturally secreted oligomers of amyloid beta protein potently inhibit hippocampal long-term potentiation in vivo. Nature 416, 535-539 (2002)

14. Shankar, G. M. et al. Amyloid-beta protein dimers isolated directly from Alzheimer's brains impair synaptic plasticity and memory. Nat. Med. 14, 837-842 (2008).

15. Li, X. et al. Novel diffusion barrier for axonal retention of Tau in neurons and its failure in neurodegeneration. EMBO J. 30, 4825-4837 (2011).

16. Cohen, T. J. et al. The acetylation of tau inhibits its function and promotes pathological tau aggregation. Nat. Commun. 2, 252 (2011).

17. Min, S. W. et al. Acetylation of tau inhibits its degradation and contributes to tauopathy. Neuron 67, 953-966 (2010).

18. Valenzuela-Fernandez, A., Cabrero, J. R., Serrador, J. M. \& Sanchez-Madrid, F. HDAC6: a key regulator of cytoskeleton, cell migration and cell-cell interactions. Trends Cell Biol. 18, 291-297 (2008).

19. Hubbert, C. et al. HDAC6 is a microtubule-associated deacetylase. Nature 417, 455-458 (2002)

20. Tapia, M., Wandosell, F. \& Garrido, J. J. Impaired function of HDAC6 slows down axonal growth and interferes with axon initial segment development. PLoS One 5, e12908 (2010).

21. Leterrier, C. et al. End-binding proteins EB3 and EB1 link microtubules to ankyrin G in the axon initial segment. Proc. Natl Acad. Sci. USA 108, 8826-8831 (2011).

22. Zilberman, Y. et al. Regulation of microtubule dynamics by inhibition of the tubulin deacetylase HDAC6. J. Cell Sci. 122, 3531-3541 (2009).

23. Petratos, S. et al. The $\beta$-amyloid protein of Alzheimer's disease increases neuronal CRMP-2 phosphorylation by a Rho-GTP mechanism. Brain 103, 90-108 (2008)

24. Yankner, B. A., Duffy, L. K. \& Kirschner, D. A. Neurotrophic and neurotoxic effects of amyloid beta protein: reversal by tachykinin neuropeptides. Science 250, 279-282 (1990).

25. Guiggiani, A. et al. Long-range and long-term interferometric tracking by static and dynamic force-clamp optical tweezers. Opt. Express 19, 22364-22376 (2011).

26. Difato, F., Pinato, G. \& Cojoc, D. Cell signaling experiments driven by optical manipulation. Int. J. Mol. Sci. 14, 8963-8984 (2013).

27. Galbraith, C. G., Yamada, K. M. \& Galbraith, J. A. Polymerizing actin fibers position integrins primed to probe for adhesion sites. Science 315, 992-995 (2007).

28. Vicente-Manzanares, M., Ma, X., Adelstein, R. S. \& Horwitz, A. R. Non-muscle myosin II takes centre stage in cell adhesion and migration. Nat. Rev. Mol. Cell Biol. 10, 778-790 (2009).
29. Yoshizaki, H. et al. Activity of Rho-family GTPases during cell division as visualized with FRET-based probes. J. Cell Biol. 162, 223-232 (2003).

30. Palazzo, A. F., Cook, T. A., Alberts, A. S. \& Gundersen, G. G. mDia mediates Rho-regulated formation and orientation of stable microtubules. Nat. Cell Biol. 3, 723-729 (2001)

31. Janke, C. \& Bulinski, J. C. Post-translational regulation of the microtubule cytoskeleton: mechanisms and functions. Nat. Rev. Mol. Cell Biol. 12, 773-786 (2011).

32. Grigoriev, I., Borisy, G. \& Vorobjev, I. Regulation of microtubule dynamics in 3T3 fibroblasts by Rho family GTPases. Cell Motil. Cytoskeleton 63, 29-40 (2006).

33. Maruta, H., Greer, K. \& Rosenbaum, J. L. The acetylation of alpha-tubulin and its relationship to the assembly and disassembly of microtubules. J. Cell Biol. 103, 571-579 (1986).

34. Matsuyama, A. et al. In vivo destabilization of dynamic microtubules by HDAC6-mediated deacetylation. EMBO J. 21, 6820-6831 (2002).

35. Zhou, D. et al. AnkyrinG is required for clustering of voltage-gated Na channels at axon initial segments and for normal action potential firing. J. Cell Biol. 143, 1295-1304 (1998).

36. Takesono, A., Heasman, S. J., Wojciak-Stothard, B., Garg, R. \& Ridley, A. J. Microtubules regulate migratory polarity through Rho/ROCK signaling in T cells. PLoS ONE 5, e8774 (2010).

37. Ridley, A. J. \& Hall, A. The small GTP-binding protein rho regulates the assembly of focal adhesions and actin stress fibers in response to growth factors Cell 70, 389-399 (1992).

38. Pertz, O., Hodgson, L., Klemke, R. L. \& Hahn, K. M. Spatiotemporal dynamics of RhoA activity in migrating cells. Nature 440, 1069-1072 (2006).

39. Hamilton, A. \& Holscher, C. The effect of ageing on neurogenesis and oxidative stress in the APP(swe)/PS1(deltaE9) mouse model of Alzheimer's disease. Brain Res. 1449, 83-93 (2012).

40. Schaefer, A. W., Kabir, N. \& Forscher, P. Filopodia and actin arcs guide the assembly and transport of two populations of microtubules with unique dynamic parameters in neuronal growth cones. J. Cell Biol. 158, 139-152 (2002).

41. Janson, M. E., de Dood, M. E. \& Dogterom, M. Dynamic instability of microtubules is regulated by force. J. Cell Biol. 161, 1029-1034 (2003).

42. Hempen, B. \& Brion, J. P. Reduction of acetylated alpha-tubulin immunoreactivity in neurofibrillary tangle-bearing neurons in Alzheimer's disease. J. Neuropathol. Exp. Neurol. 55, 964-972 (1996).

43. Perez, M. et al. Tau-an inhibitor of deacetylase HDAC6 function. J. Neurochem. 109, 1756-1766 (2009).

44. Chuang, D. M., Leng, Y., Marinova, Z., Kim, H. J. \& Chiu, C. T. Multiple roles of HDAC inhibition in neurodegenerative conditions. Trends Neurosci. 32, 591-601 (2009).

45. Selenica, M. L. et al. Histone deacetylase 6 inhibition improves memory and reduces total tau levels in a mouse model of tau deposition. Alzheimers Res. Ther. 6, 12 (2014).

46. Govindarajan, N. et al. Reducing HDAC6 ameliorates cognitive deficits in a mouse model for Alzheimer's disease. EMBO Mol. Med. 5, 52-63 (2012).

47. d'Ydewalle, C. et al. HDAC6 inhibitors reverse axonal loss in a mouse model of mutant HSPB1-induced Charcot-Marie-Tooth disease. Nat. Med. 17, 968-974 (2011).

48. Dompierre, J. P. et al. Histone deacetylase 6 inhibition compensates for the transport deficit in Huntington's disease by increasing tubulin acetylation J. Neurosci. 27, 3571-3583 (2007).

49. Bobrowska, A., Paganetti, P., Matthias, P. \& Bates, G. P. Hdac6 knock-out increases tubulin acetylation but does not modify disease progression in the R6/ 2 mouse model of Huntington's disease. PLoS ONE 6, e20696 (2011).

50. Watabe, M. \& Nakaki, T. Protein kinase CK2 regulates the formation and clearance of aggresomes in response to stress. J. Cell Sci. 124, 1519-1532 (2011).

51. Iwata, A., Riley, B. E., Johnston, J. A. \& Kopito, R. R. HDAC6 and microtubules are required for autophagic degradation of aggregated huntingtin. J. Biol. Chem. 280, 40282-40292 (2005).

52. Pandey, U. B. et al. HDAC6 rescues neurodegeneration and provides an essential link between autophagy and the UPS. Nature 447, 859-863 (2007).

53. Du, G. et al. Drosophila histone deacetylase 6 protects dopaminergic neurons against $\{$ alpha\}-synuclein toxicity by promoting inclusion formation. Mol. Biol Cell 21, 2128-2137 (2010).

54. Ageta-Ishihara, N. et al. Septins promote dendrite and axon development by negatively regulating microtubule stability via HDAC6-mediated deacetylation. Nat. Commun. 4, 2532 (2013).

55. Fiesel, F. C., Schurr, C., Weber, S. S. \& Kahle, P. J. TDP-43 knockdown impairs neurite outgrowth dependent on its target histone deacetylase 6. Mol. Neurodegener. 6, 64 (2011).

56. Ren, Y., Zhao, J. \& Feng, J. Parkin binds to alpha/beta tubulin and increases their ubiquitination and degradation. J. Neurosci. 23, 3316-3324 (2003). 
57. Ding, H., Dolan, P. J. \& Johnson, G. V. Histone deacetylase 6 interacts with the microtubule-associated protein tau. J. Neurochem. 106, 2119-2130 (2008).

58. Hoover, B. R. et al. Tau mislocalization to dendritic spines mediates synaptic dysfunction independently of neurodegeneration. Neuron 68, 1067-1081 (2010).

59. Noack, M., Leyk, J. \& Richter-Landsberg, C. HDAC6 inhibition results in tau acetylation and modulates tau phosphorylation and degradation in oligodendrocytes. Glia 62, 535-547 (2014).

60. Elbaum-Garfinkle, S., Cobb, G., Compton, J. T., Li, X. H. \& Rhoades, E. Tau mutants bind tubulin heterodimers with enhanced affinity. Proc. Natl Acad. Sci. USA 111, 6311-6316 (2014).

61. Takemura, R. et al. Increased microtubule stability and alpha tubulin acetylation in cells transfected with microtubule-associated proteins MAP1B, MAP2 or tau. J. Cell Sci. 103, 953-964 (1992).

62. Bradke, F. \& Dotti, C. G. The role of local actin instability in axon formation. Science 283, 1931-1934 (1999).

63. Witte, H., Neukirchen, D. \& Bradke, F. Microtubule stabilization specifies initial neuronal polarization. J. Cell Biol. 180, 619-632 (2008).

64. Gallo, G., Yee, Jr. H. F. \& Letourneau, P. C. Actin turnover is required to prevent axon retraction driven by endogenous actomyosin contractility. J. Cell Biol. 158, 1219-1228 (2002).

65. Fulga, T. A. et al. Abnormal bundling and accumulation of F-actin mediates tau-induced neuronal degeneration in vivo. Nat. Cell Biol. 9, 139-148 (2007).

66. Sun, X. et al. Selective filtering defect at the axon initial segment in Alzheimer's disease mouse models. Proc. Natl Acad. Sci. USA 111, 14271-14276 (2014)

67. Aviv, M. S. et al. Motility flow and growth-cone navigation analysis during in vitro neuronal development by long-term bright-field imaging. J. Biomed. Opt. 18, 111415 (2013).
68. Shahapure, R. et al. Force generation in lamellipodia is a probabilistic process with fast growth and retraction events. Biophys. J. 98, 979-988 (2010).

\section{Acknowledgements}

We thank G. Scita (IFOM, Milan, Italy) for GFP-LifeAct plasmid and M. Matsuda (Kyoto University, Kyoto, Japan) for Raichu plasmid. We would like to thank T. Nieus for critical discussion on the analysis and interpretation of force spectroscopy data and C. Canale for assistance on AFM data. We also would like to thank D. Cojoc for insightful discussion on optical tweezers experiments.

\section{Author contributions}

H.T. conducted most of the experiments. M.E. and A.E. performed the experiments and data analysis during the review process. A.P. carried out the electrophysiology experiments. F.D. carried out the experiments of force spectroscopy and optical tweezers. H.T. A.P., A.B., M.V. and F.D. analysed the data. H.T., F.D. and E.C. conceived the project and wrote the manuscript. E.C. secured funding and supervised the experiments.

\section{Additional information}

Supplementary Information accompanies this paper at http://www.nature.com/ naturecommunications

Competing financial interests: The authors declare no competing financial interests

Reprints and permission information is available online at http://npg.nature.com/ reprintsandpermissions/

How to cite this article: Tsushima, H. et al. HDAC6 and RhoA are novel players in Abeta-driven disruption of neuronal polarity. Nat. Commun. 6:7781 doi: $10.1038 /$ ncomms8781 (2015). 